\title{
Non-divergence form parabolic equations associated with non-commuting vector fields: boundary behavior of nonnegative solutions
}

\author{
Marie Frentz, Nicola Garofalo, Elin Götmark, Isidro Munive \\ AND KAJ NYSTRÖM
}

Abstract. In a cylinder $\Omega_{T}=\Omega \times(0, T) \subset \mathbb{R}_{+}^{n+1}$ we study the boundary behavior of nonnegative solutions of second order parabolic equations of the form

$$
H u=\sum_{i, j=1}^{m} a_{i j}(x, t) X_{i} X_{j} u-\partial_{t} u=0,(x, t) \in \mathbb{R}_{+}^{n+1},
$$

where $X=\left\{X_{1}, \ldots, X_{m}\right\}$ is a system of $C^{\infty}$ vector fields in $\mathbb{R}^{n}$ satisfying Hörmander's rank condition (1.2), and $\Omega$ is a non-tangentially accessible domain with respect to the Carnot-Carathéodory distance $d$ induced by $X$. Concerning the matrix-valued function $A=\left\{a_{i j}\right\}$, we assume that it is real, symmetric and uniformly positive definite. Furthermore, we suppose that its entries $a_{i j}$ are Hölder continuous with respect to the parabolic distance associated with $d$. Our main results are: 1) a backward Harnack inequality for nonnegative solutions vanishing on the lateral boundary (Theorem 1.1);2) the Hölder continuity up to the boundary of the quotient of two nonnegative solutions which vanish continuously on a portion of the lateral boundary (Theorem 1.2);3) the doubling property for the parabolic measure associated with the operator $H$ (Theorem 1.3). These results generalize to the subelliptic setting of the present paper, those in Lipschitz cylinders by Fabes, Safonov and Yuan in [20,39]. With one proviso: in those papers the authors assume that the coefficients $a_{i j}$ be only bounded and measurable, whereas we assume Hölder continuity with respect to the intrinsic parabolic distance.

Mathematics Subject Classification (2010): 31C05 (primary); 35C15, 65N99 (secondary).

\section{Introduction}

Let $\Omega \subset \mathbb{R}^{n}$ be a bounded domain and consider the cylinder $\Omega_{T}=\Omega \times(0, T) \subset$ $\mathbb{R}_{+}^{n+1}$, where $T>0$ is fixed. In this paper we establish a number of results concern-

Second author supported in part by NSF Grant DMS-07010001.

Fourth author supported in part by the second author's NSF Grant DMS-07010001.

Received July 21, 2010; accepted January 13, 2011. 
ing the boundary behavior of nonnegative solutions in $\Omega_{T}$ of second order parabolic equations of the type

$$
H u=L u-\partial_{t} u=\sum_{i, j=1}^{m} a_{i j}(x, t) X_{i} X_{j} u-\partial_{t} u=0 .
$$

Here, $X=\left\{X_{1}, \ldots, X_{m}\right\}$ is a system of $C^{\infty}$ vector fields in $\mathbb{R}^{n}$ satisfying Hörmander's rank condition, see [25]:

$$
\operatorname{rank} \operatorname{Lie}\left[X_{1}, \ldots, X_{m}\right] \equiv n \text {. }
$$

Concerning the $m \times m$ matrix-valued function $A(x, t)=\left\{a_{i j}(x, t)\right\}$ we assume that it is symmetric, with bounded and measurable entries, and that there exists $\lambda \in[1, \infty)$ such that for every $(x, t) \in \mathbb{R}^{n+1}$, and $\xi \in \mathbb{R}^{m}$,

$$
\lambda^{-1}|\xi|^{2} \leq \sum_{i, j=1}^{m} a_{i j}(x, t) \xi_{i} \xi_{j} \leq \lambda|\xi|^{2}
$$

When $m=n$ and $\left\{X_{1}, \ldots, X_{m}\right\}=\left\{\partial_{x_{1}}, \ldots, \partial_{x_{n}}\right\}$, the operator $H$ in (1.1) coincides with that studied in $[20,39]$. However, in contrast with these papers, in which the coefficients were assumed only bounded and measurable, we will also assume that the entries of the matrix $A(x, t)$ are Hölder continuous with respect to the intrinsic parabolic distance associated with the system $X$. More precisely, we indicate with $d(x, y)$ the Carnot-Carathéodory distance, between $x, y \in \mathbb{R}^{n}$, induced by $\left\{X_{1}, \ldots, X_{m}\right\}$. We let

$$
d_{p}(x, t, y, s)=\left(d(x, y)^{2}+|t-s|\right)^{1 / 2}
$$

denote the parabolic distance associated with the metric $d$. Then, we assume that there exist $C>0$, and $\sigma \in(0,1)$, such that for $(x, t),(y, s) \in \mathbb{R}^{n+1}$,

$$
\left|a_{i j}(x, t)-a_{i j}(y, s)\right| \leq C d_{p}(x, t, y, s)^{\sigma}, \quad i, j \in\{1, . ., m\} .
$$

The reason for imposing (1.4) will be discussed below.

Concerning the domain $\Omega$ we will assume that $\Omega$ is a NTA domain (nontangentially accessible domain), with parameters $M, r_{0}$, in the sense of $[7,11]$, see Definition 2.6 below. Under this assumption we can prove that all points on the parabolic boundary

$$
\partial_{p} \Omega_{T}=S_{T} \cup(\Omega \times\{0\}), \quad S_{T}=\partial \Omega \times(0, T),
$$

of the cylinder $\Omega_{T}$ are regular for the Dirichlet problem for the operator $H$ in (1.1). In particular, for any $f \in C\left(\partial_{p} \Omega_{T}\right)$, there exists a unique Perron-Wiener-BrelotBauer solution $u=u_{f}^{\Omega_{T}} \in C\left(\bar{\Omega}_{T}\right)$ to the Dirichlet problem

$$
H u=0 \text { in } \Omega_{T}, \quad u=f \text { on } \partial_{p} \Omega_{T} .
$$


Moreover, one can conclude that for every $(x, t) \in \Omega_{T}$ there exists a unique probability measure $d \omega^{(x, t)}$ on $\partial_{p} \Omega_{T}$ such that

$$
u(x, t)=\int_{\partial_{p} \Omega_{T}} f(y, s) d \omega^{(x, t)}(y, s) .
$$

Henceforth, we refer to $\omega^{(x, t)}$ as the $H$-parabolic measure relative to $(x, t)$ and $\Omega_{T}$.

The metric ball centered at $x \in \mathbb{R}^{n}$ with radius $r>0$ will be indicated with

$$
B_{d}(x, r)=\left\{y \in \mathbb{R}^{n}: d(x, y)<r\right\} .
$$

For $(x, t) \in \mathbb{R}^{n+1}$ and $r>0$ we let

$$
C_{r}^{-}(x, t)=B_{d}(x, r) \times\left(t-r^{2}, t\right), \quad C_{r}(x, t)=B_{d}(x, r) \times\left(t-r^{2}, t+r^{2}\right),
$$

and we define

$$
\Delta(x, t, r)=S_{T} \cap C_{r}(x, t) .
$$

By Definition 2.6 below, if $\Omega$ is a given NTA domain with parameters $M$ and $r_{0}$, then for any $x_{0} \in \partial \Omega, 0<r<r_{0}$, there exists a non-tangential corkscrew, i.e., a point $A_{r}\left(x_{0}\right) \in \Omega$, such that

$$
M^{-1} r<d\left(x_{0}, A_{r}\left(x_{0}\right)\right)<r, \text { and } d\left(A_{r}\left(x_{0}\right), \partial \Omega\right) \geq M^{-1} r .
$$

In the following we let $A_{r}\left(x_{0}, t_{0}\right)=\left(A_{r}\left(x_{0}\right), t_{0}\right)$ whenever $\left(x_{0}, t_{0}\right) \in S_{T}$ and $0<$ $r<r_{0}$. When we say that a constant $c$ depends on the operator $H$ we mean that $c$ depends on the dimension $n$, the number of vector fields $m$, the vector fields $\left\{X_{1}, \ldots, X_{m}\right\}$, the constant $\lambda$ in (1.3) and the parameters $C, \sigma$ in (1.4). We let $\operatorname{diam}(\Omega)=\sup \{d(x, y) \mid x, y \in \Omega\}$ denote the diameter of $\Omega$. The following theorems represent the main results of this paper.

Theorem 1.1 (Backward Harnack inequality). Let $u$ be a nonnegative solution of $H u=0$ in $\Omega_{T}$ vanishing continuously on $S_{T}$. Let $0<\delta \ll \sqrt{T}$ be a fixed constant, let $\left(x_{0}, t_{0}\right) \in S_{T}, \delta^{2} \leq t_{0} \leq T-\delta^{2}$, and assume that $r<$ $\min \left\{r_{0} / 2, \sqrt{\left(T-t_{0}-\delta^{2}\right) / 4}, \sqrt{\left(t_{0}-\delta^{2}\right) / 4}\right\}$. Then, there exists a constant $c=$ $c\left(H, M, r_{0}, \operatorname{diam}(\Omega), T, \delta\right), 1 \leq c<\infty$, such that for every $(x, t) \in \Omega_{T} \cap$ $C_{r / 4}\left(x_{0}, t_{0}\right)$ one has

$$
u(x, t) \leq c u\left(A_{r}\left(x_{0}, t_{0}\right)\right) .
$$

Theorem 1.2 (Boundary Hölder continuity of quotients of solutions). Let $u, v$ be nonnegative solutions of $H u=0$ in $\Omega_{T}$. Given $\left(x_{0}, t_{0}\right) \in S_{T}$, assume that $r<$ $\min \left\{r_{0} / 2, \sqrt{\left(T-t_{0}\right) / 4}, \sqrt{t_{0} / 4}\right\}$. If $u, v$ vanish continuously on $\Delta\left(x_{0}, t_{0}, 2 r\right)$, then the quotient $v / u$ is Hölder continuous on the closure of $\Omega_{T} \cap C_{r}^{-}\left(x_{0}, t_{0}\right)$. 
Theorem 1.3 (Doubling property of the $H$-parabolic measure). Let $K \geq 100$ and $v \in(0,1)$ be fixed constants. Let $\left(x_{0}, t_{0}\right) \in S_{T}$, and suppose that $r<$ $\min \left\{v r_{0} / 2, \sqrt{\left(T-t_{0}\right) / 4}, \sqrt{t_{0} / 4}\right\}$. Then, there exists a constant $c=c\left(H, M, v, K, r_{0}\right)$, $1 \leq c<\infty$, such that for every $(x, t) \in \Omega_{T}$, with $d\left(x_{0}, x\right) \leq K\left|t-t_{0}\right|^{1 / 2}$, $t-t_{0} \geq 16 r^{2}$, one has

$$
\omega^{(x, t)}\left(\Delta\left(x_{0}, t_{0}, 2 r\right)\right) \leq c \omega^{(x, t)}\left(\Delta\left(x_{0}, t_{0}, r\right)\right) .
$$

Concerning Theorems 1.1, 1.2 and 1.3, we note that the study of the type of problems considered in this paper has a long and rich history which, for uniformly parabolic equations in $\mathbb{R}^{n+1}$ (i.e., when in (1.1) one has $m=n$ and $\left\{X_{1}, \ldots, X_{m}\right\}=$ $\left.\left\{\partial_{x_{1}}, \ldots, \partial_{x_{n}}\right\}\right)$, culminated with the celebrated papers of Fabes, Safonov and Yuan $[19,20,39]$. In these works the authors proved Theorem 1.1-1.3 for uniformly parabolic equations, both in divergence and non-divergence form, whose coefficients are only bounded and measurable. We remark that, while these authors work in Lipschitz cylinders, one can easily see that their proofs can be generalized to the setting of bounded NTA domains in the sense of [26]. While the works $[20,39]$ completed this line of research for parabolic operators in non-divergence form, prior contributions by other researchers are contained in [17, 21, 22, 29]. For the corresponding developments for second order parabolic operators in divergence form we refer to $[16,19,34]$. For the elliptic theory, for both operators in divergence and nondivergence form, we refer to $[1,6,15,26]$. Finally, and for completion, we also note that second order elliptic and parabolic operators in divergence form with singular lower order terms were studied in [24,27].

In the subelliptic setting of the present paper, i.e., when $m<n$ and $X=$ $\left\{X_{1}, \ldots, X_{m}\right\}$ is assumed to satisfy (1.2), much less is known. Several delicate new issues arise in connection with the intricate (sub-Riemannian) geometry associated with the vector fields, and the interplay of such geometry with the socalled characteristic points on the boundary of the relevant domain. In addition, the derivatives along the vector fields do not commute, and the commutators are effectively derivatives of higher order. For all these aspects we refer the reader to the works $[7,10,11,13,14,30-32,35]$, but this only represents a partial list of references.

In the stationary case, and for operators in divergence form, results similar to those in the present paper have been obtained in $[7,10,11]$, see also [8,9], whereas for parabolic operators in divergence form the reader is referred to the recent paper by one of us [33]. The methods in [33], however, extensively exploit the divergence structure of the operator and do not apply to the setting of the present paper.

We stress that for non-divergence form operators such as those treated in this paper, results such as Theorems 1.1-1.3 are new even for the case of stationary equations such as

$$
L u=\sum_{i, j=1}^{m} a_{i j}(x) X_{i} X_{j} u=0
$$


In view of these considerations our paper provides a novel contribution to the understanding of the boundary behavior of solutions to parabolic equations arising from a system of non-commuting vector fields.

Concerning the proofs of Theorems 1.1-1.3 our approach is modeled on the ideas developed by Fabes, Safonov and Yuan in [20,39]. In fact, the ideas in those papers have provided an important guiding line for our work. Yet, the arguments in $[20,39]$ use mainly elementary principles like comparison principles, interior regularity theory, the (interior) Harnack inequality, Hölder continuity type estimates and decay estimates at the lateral boundary, for solutions which vanish on a portion of the lateral boundary, as well as estimates for the Cauchy problem and the fundamental solution associated to the operator at hand. In this connection it is important that the reader keep in mind that when the matrix $A(x, t)=\left\{a_{i j}(x, t)\right\}$ in (1.1) has entries which are just bounded and measurable, then most of these results presently represent in our setting terra incognita. More specifically, the counterparts of the Harnack inequality of Krylov and Safonov [29] and the Alexandrov-Bakel'manPucci type maximum principle due to Krylov [28] presently constitute fundamental open questions.

With this being said, our work uses heavily the recent important results of Bramanti, Brandolini, Lanconelli and Uguzzoni [5], see also [4], concerning the (interior) Harnack inequality, the Cauchy problem and the existence and Gaussian estimates for fundamental solutions for the non-divergence form operators $H$ defined in (1.1). In fact, we assume (1.4) precisely in order to be able to use results from [5]. We want to stress, however, that we have strived throughout the whole paper to provide proofs which are "purely metrical". By this we mean that, should the above mentioned counterpart of the results in $[28,29]$ become available, then our proofs would carry to the more general setting of bounded and measurable coefficients in (1.1) with minor changes.

In closing we mention that the rest of the paper is organized as follows. Section 2 is of a preliminary nature. In it we collect some notation and results concerning basic underlying principles, and we also introduce the notion of NTA domains following [7]. In Section 3 we prove a number of basic estimates concerning the boundary behavior of nonnegative solutions of (1.1). In addition we prove a number of technical lemmas which allow us to present the proofs of Theorems 1.1-1.3 in a quite condensed manner. Finally, the proofs of Theorems 1.1 and 1.2 will be presented in Section 4, whereas that of Theorem 1.3 will be given in Section 5.

\section{Preliminaries}

In this section we introduce some notation and state a number of preliminary results for the operator $H$ defined in (1.1). Specifically, we will discuss the Cauchy problem and Gaussian estimates for the fundamental solution, the Harnack inequality and comparison principle, and the Dirichlet problem in bounded domains. In particular, we also justify the notion of $H$-parabolic measure and introduce the notion of NTA domain. 


\subsection{Notation}

In $\mathbb{R}^{n}$, with $n \geq 3$, we consider a system $X=\left\{X_{1}, \ldots, X_{m}\right\}$ of $C^{\infty}$ vector fields satisfying Hörmander's rank condition (1.2). As in [18], a piecewise $C^{1}$ curve $\gamma:[0, \ell] \rightarrow \mathbb{R}^{n}$ is called subunitary if at every $t \in[0, \ell]$ at which $\gamma^{\prime}(t)$ exists one has for every $\xi \in \mathbb{R}^{n}$

$$
<\gamma^{\prime}(t), \xi>^{2} \leq \sum_{j=1}^{m}<X_{j}(\gamma(t)), \xi>^{2}
$$

We note explicitly that the above inequality forces $\gamma^{\prime}(t)$ to belong to the span of $\left\{X_{1}(\gamma(t)), \ldots, X_{m}(\gamma(t))\right\}$. The subunit length of $\gamma$ is by definition $l_{s}(\gamma)=\ell$. If we fix an open set $\Omega \subset \mathbb{R}^{n}$, then given $x, y \in \Omega$, denote by $\mathcal{S}_{\Omega}(x, y)$ the collection of all subunitary $\gamma:[0, \ell] \rightarrow \Omega$ which join $x$ to $y$. The accessibility theorem of Chow and Rashevsky, [12,37], states that, if $\Omega$ is connected, then for every $x, y \in \Omega$ there exists $\gamma \in \mathcal{S}_{\Omega}(x, y)$. As a consequence, if we define

$$
d_{\Omega}(x, y)=\inf \left\{l_{s}(\gamma) \mid \gamma \in \mathcal{S}_{\Omega}(x, y)\right\},
$$

we obtain a distance on $\Omega$, called the Carnot-Carathéodory distance, associated with the system $X$. When $\Omega=\mathbb{R}^{n}$, we write $d(x, y)$ instead of $d_{\mathbb{R}^{n}}(x, y)$. It is clear that $d(x, y) \leq d_{\Omega}(x, y), x, y \in \Omega$, for every connected open set $\Omega \subset \mathbb{R}^{n}$. In [36] it was proved that, given $\Omega \subset \subset \mathbb{R}^{n}$, there exist $C, \epsilon>0$ such that

$$
C|x-y| \leq d_{\Omega}(x, y) \leq C^{-1}|x-y|^{\epsilon}, \quad x, y \in \Omega .
$$

This gives $d(x, y) \leq C^{-1}|x-y|^{\epsilon}, x, y \in \Omega$, and therefore

$$
i:\left(\mathbb{R}^{n},|\cdot|\right) \rightarrow\left(\mathbb{R}^{n}, d\right) \quad \text { is continuous. }
$$

Furthermore, it is easy to see that also the continuity of the opposite inclusion holds [23], and therefore the metric and the Euclidean topologies are equivalent.

For $x \in \mathbb{R}^{n}$ and $r>0$, we let $B_{d}(x, r)=\left\{y \in \mathbb{R}^{n} \mid d(x, y)<r\right\}$. The basic properties of these balls were established by Nagel, Stein and Wainger in their seminal paper [36]. These authors proved in particular that, given bounded open set $U \subset \mathbb{R}^{n}$, there exist constants $C, R_{0}>0$ such that, for any $x \in U$, and $0<r \leq R_{0}$,

$$
C \leq \frac{B_{d}(x, r)}{\Lambda(x, r)} \leq C^{-1}
$$

where $\Lambda(x, r)=\sum_{I}\left|a_{I}(x)\right| r^{d_{I}}$ is a polynomial function with continuous coefficients. As a consequence, one has with $C_{1}>0$,

$$
\left|B_{d}(x, 2 r)\right| \leq C_{1}\left|B_{d}(x, r)\right| \quad \text { for every } \quad x \in U \quad \text { and } \quad 0<r \leq R_{0} \text {. }
$$


In what follows, given $\beta \in(0,1)$, we let $\Gamma^{\beta}\left(\Omega_{T}\right)$ denote the space of functions $u: \Omega_{T} \rightarrow \mathbb{R}$ such that

$$
\begin{aligned}
\|u\|_{\Gamma^{\beta}\left(\Omega_{T}\right)}:= & \sup _{\Omega_{T}}|u| \\
& +\sup _{(x, t),\left(x^{\prime}, t^{\prime}\right) \in \Omega_{T},(x, t) \neq\left(x^{\prime}, t^{\prime}\right)} \frac{\left|u(x, t)-u\left(x^{\prime}, t^{\prime}\right)\right|}{d_{p}\left(x, t, x^{\prime}, t^{\prime}\right)^{\beta}}<\infty .
\end{aligned}
$$

We say that $u$ has a Lie derivative along $X_{j}$, at $(x, t) \in \Omega_{T}$, if $u \circ \gamma$ is differentiable at 0 , where $\gamma$ is the integral curve of $X_{j}$ such that $\gamma(0)=(x, t)$. Moreover, we indicate with $\Gamma^{2+\beta}\left(\Omega_{T}\right)$ the space of functions $u \in \Gamma^{\beta}\left(\Omega_{T}\right)$ which admit Lie derivatives up to second order along $X_{1}, \ldots, X_{m}$, and up to order one with respect to $t$, in $\Gamma^{\beta}\left(\Omega_{T}\right)$. If $u \in \Gamma^{2+\beta}\left(\Omega_{T}\right)$ then we let $\|u\|_{\Gamma^{2+\beta}\left(\Omega_{T}\right)}$ denote the naturally defined norm of $u$. Furthermore, $u \in \Gamma_{\mathrm{loc}}^{\beta}\left(\Omega_{T}\right)$ if $u \in \Gamma^{\beta}(D)$ for any compact subset $D$ of $\Omega_{T}$. The space $\Gamma_{\text {loc }}^{2+\beta}\left(\Omega_{T}\right)$ is defined analogously. Finally, if $\beta=0$ then we simply write $\Gamma^{2}\left(\Omega_{T}\right)$ for $\Gamma^{2+0}\left(\Omega_{T}\right)$. Throughout the paper we will use the following notation:

$$
\begin{aligned}
C_{r}(x, t) & =B_{d}(x, r) \times\left(t-r^{2}, t+r^{2}\right), \\
C_{r}^{+}(x, t) & =B_{d}(x, r) \times\left(t, t+r^{2}\right), \\
C_{r}^{-}(x, t) & =B_{d}(x, r) \times\left(t-r^{2}, t\right), \\
C_{r_{1}, r_{2}}(x, t) & =B_{d}\left(x, r_{1}\right) \times\left(t-r_{2}^{2}, t+r_{2}^{2}\right), \\
C_{r_{1}, r_{2}}^{+}(x, t) & =B_{d}\left(x, r_{1}\right) \times\left(t, t+r_{2}^{2}\right), \\
C_{r_{1}, r_{2}}^{-}(x, t) & =B_{d}\left(x, r_{1}\right) \times\left(t-r_{2}^{2}, t\right),
\end{aligned}
$$

for $(x, t) \in \mathbb{R}^{n+1}$ and $r, r_{1}, r_{2}>0$. Furthermore, if $\Omega \subset \mathbb{R}^{n}$ is a bounded domain, and $T>0$ and $\delta>0$ are given, then we let

$$
\Omega^{\delta}=\{x \in \Omega \mid d(x, \partial \Omega)>\delta\}, \Omega_{T}^{\delta}=\Omega^{\delta} \times(0, T) .
$$

\subsection{The Cauchy problem}

Let $H$ be defined as in (1.1), with the hypothesis (1.2), (1.3) and (1.4) in place. These assumptions allow us to use some basic results established in [5]. In particular, for what concerns the existence of a fundamental solution of the operator $H$, and Gaussian estimates, we will henceforth suppose, as it is done in [5], that the sub-Laplacian $\sum_{i=1}^{m} X_{i}^{2}$ associated with $X$ coincides with the standard Laplacian $\Delta=\sum_{j=1}^{n} \partial_{x_{j}}^{2}$ in $\mathbb{R}^{n}$ outside of a fixed compact set in $\mathbb{R}^{n}$.

In [5] it is proved that, under such hypothesis, there exists a fundamental solution, $\Gamma$, for $H$, with a number of important properties. In particular, $\Gamma$ is a continuous function away from the diagonal of $\mathbb{R}^{n+1} \times \mathbb{R}^{n+1}$ and $\Gamma(x, t, \xi, \tau)=0$ for $t \leq$ 
$\tau$. Moreover, $\Gamma(\cdot, \cdot, \xi, \tau) \in \Gamma_{\text {loc }}^{2+\alpha}\left(\mathbb{R}^{n+1} \backslash\{(\xi, \tau)\}\right)$ for every fixed $(\xi, \tau) \in \mathbb{R}^{n+1}$ and $H(\Gamma(\cdot, \cdot, \xi, \tau))=0$ in $\mathbb{R}^{n+1} \backslash\{(\xi, \tau)\}$. For every $\psi \in C_{0}^{\infty}\left(\mathbb{R}^{n+1}\right)$ the function

$$
w(x, t)=\int_{\mathbb{R}^{n+1}} \Gamma(x, t, \xi, \tau) \psi(\xi, \tau) d \xi d \tau
$$

belongs to $\Gamma_{\text {loc }}^{2+\alpha}\left(\mathbb{R}^{n+1}\right)$ and we have $H w=\psi$ in $\mathbb{R}^{n+1}$. Furthermore, let $\mu \geq 0$ and $T_{2}>T_{1}$ be such that $\left(T_{2}-T_{1}\right) \mu$ is small enough, let $0<\beta \leq \alpha$, let $g \in C^{0, \beta}\left(\mathbb{R}^{n} \times\right.$ $\left.\left[T_{1}, T_{2}\right]\right)$ and $f \in C\left(\mathbb{R}^{n}\right)$ be such that $|g(x, t)|,|f(x)| \leq c \exp \left(\mu d(x, 0)^{2}\right)$ for some constant $c>0$. Then, for $x \in \mathbb{R}^{n}, t \in\left(T_{1}, T_{2}\right]$, the function

$$
u(x, t)=\int_{\mathbf{R}^{n}} \Gamma\left(x, t, \xi, T_{1}\right) f(\xi) d \xi+\int_{T_{1}}^{t} \int_{\mathbf{R}^{n}} \Gamma(x, t, \xi, \tau) g(\xi, \tau) d \xi d \tau
$$

belongs to the class $\Gamma_{\text {loc }}^{2+\beta}\left(\mathbb{R}^{n} \times\left(T_{1}, T_{2}\right)\right) \cap C\left(\mathbb{R}^{n} \times\left[T_{1}, T_{2}\right]\right)$. Moreover, $u$ solves the Cauchy problem

$$
H u=g \text { in } \mathbb{R}^{n} \times\left(T_{1}, T_{2}\right), u\left(\cdot, T_{1}\right)=f(\cdot) \text { in } \mathbb{R}^{n} .
$$

One also has the following Gaussian bounds.

Lemma 2.1. There exist a positive constant $C$ and, for every $T>0$, a positive constant $c=c(T)$ such that, if $0<t-\tau \leq T, x, \xi \in \mathbb{R}^{n}$, then

$$
c^{-1} \frac{e^{-C d(x, \xi)^{2} /(t-\tau)}}{|B(x, \sqrt{t-\tau})|} \leq \Gamma(x, t, \xi, \tau) \leq c \frac{e^{-C^{-1} d(x, \xi)^{2} /(t-\tau)}}{|B(x, \sqrt{t-\tau})|} .
$$

Furthermore, one also has

$$
\left|X_{i} \Gamma(\cdot, t, \xi, \tau)(x)\right| \leq c(t-\tau)^{-1 / 2} \frac{e^{-C^{-1} d(x, \xi)^{2} /(t-\tau)}}{|B(x, \sqrt{t-\tau})|},
$$

and

$$
\left|X_{i} X_{j} \Gamma(\cdot, t, \xi, \tau)(x)\right|+\left|\partial_{t} \Gamma(x, \cdot, \xi, \tau)(t)\right| \leq c(t-\tau)^{-1} c \frac{e^{-C^{-1} d(x, \xi)^{2} /(t-\tau)}}{|B(x, \sqrt{t-\tau})|}
$$

\subsection{The Harnack inequality and strong maximum principle}

We next state the Harnack inequality and the strong maximum principle for the operator $H$, see [4] and also [5]. 
Theorem 2.2. Let $R>0,0<h_{1}<h_{2}<1$ and $\gamma \in(0,1)$. Then, there exists a positive constant $C=C\left(h_{1}, h_{2}, \gamma, R\right)$ such that the following holds for every $(\xi, \tau) \in \mathbb{R}^{n+1}, r \in(0, R]$. If

$$
u \in \Gamma^{2}\left(C_{r}^{-}(\xi, \tau)\right) \cap C\left(\overline{C_{r}^{-}(\xi, \tau)}\right)
$$

satisfies $H u=0, u \geq 0$, in $C_{r}^{-}(\xi, \tau)$, then

$$
u(x, t) \leq C u(\xi, \tau) \text { whenever }(x, t) \in \overline{C_{\gamma r, h_{2} r}^{-}(\xi, \tau)} \backslash C_{\gamma r, h_{1} r}^{-}(\xi, \tau) .
$$

Theorem 2.3. Let $\Omega \subset \mathbb{R}^{n}$ be a connected, bounded open set, and let $T>0$. Let $u \in \Gamma^{2}\left(\Omega_{T}\right)$ and assume that $L u \geq 0, u \leq 0$ in $\Omega_{T}$. Assume that $u\left(x_{0}, t_{0}\right)=0$ for some $\left(x_{0}, t_{0}\right) \in \Omega_{T}$. Then $u(x, t) \equiv 0$ whenever $(x, t) \in \Omega_{T} \cap\left\{t: t \leq t_{0}\right\}$.

\subsection{The Dirichlet problem}

In the following we let $D$ be any bounded open subset of $\mathbb{R}^{n+1}$ and we study the Dirichlet problem

$$
H u=0 \text { in } D, u=f \text { on } \partial_{p} D,
$$

with $f \in C\left(\partial_{p} D\right)$. Here, $\partial_{p} D$ denotes the parabolic boundary of $D$. If $u: D \rightarrow \mathbb{R}$ is a smooth function satisfying $H u=0$ in $D$, then we say that $u$ is $H$-parabolic in $D$. We denote by $P(D)$ the linear space of functions which are $H$-parabolic in $D$.

We say that $D$ is $H$-regular if for any $f \in C\left(\partial_{p} D\right)$ there exists a unique function $H_{f}^{D} \in P(D)$ such that $\lim _{(x, t) \rightarrow\left(x_{0}, t_{0}\right)} H_{f}^{D}(x, t)=f\left(x_{0}, t_{0}\right)$ for every $\left(x_{0}, t_{0}\right) \in \partial_{p} D$. Following the arguments in [30], see in particular Theorems 6.5 and 10.1 , we can easily construct a basis for the Euclidean topology of $\mathbb{R}^{n+1}$ which is made of cylindrical $H$-regular sets. Furthermore, if $D$ is $H$-regular, then in view of Theorem 2.3 (one actually only needs the weak maximum principle) for every fixed $(x, t) \in D$ the map $f \mapsto H_{f}^{D}(x, t)$ defines a positive linear functional on $C\left(\partial_{p} D\right)$. By the Riesz representation theorem there exists a unique Borel measure $\omega=\omega_{D}$, supported in $\partial_{p} D$, such that

$$
H_{f}^{D}(x, t)=\int_{\partial_{p} D} f(y, s) d \omega^{(x, t)}(y, s), \quad \text { for every } f \in C\left(\partial_{p} D\right) .
$$

We will refer to $\omega^{(x, t)}=\omega_{D}^{(x, t)}$ as the $H$-parabolic measure relative to $D$ and $(x, t)$.

A lower semi-continuous function $u: D \rightarrow]-\infty, \infty]$ is said to be $H$ superparabolic in $D$ if $u<\infty$ in a dense subset of $D$ and if

$$
u(x, t) \geq \int_{\partial V} u(y, s) d \omega_{V}^{(x, t)}(y, s),
$$

for every open $H$-regular set $V \subset \bar{V} \subset D$ and for every $(x, t) \in V$. We denote by $\bar{S}(D)$ the set of $H$-superparabolic functions in $D$, and by $\bar{S}^{+}(D)$ the set of the 
functions in $\bar{S}(D)$ which are nonnegative. A function $v: D \rightarrow[-\infty, \infty$ [ is said to be $H$-subparabolic in $D$ if $-v \in \bar{S}(D)$ and we write $S(D):=-\bar{S}(D)$. As the collection of $H$-regular sets is a basis for the Euclidean topology, it follows that $\bar{S}(D) \cap \underline{S}(D)=P(D)$. Finally, we recall that $H_{f}^{D}$ can be realized as the generalized solution in the sense of Perron-Wiener-Brelot-Bauer to the problem in (2.11). In particular,

$$
\inf \overline{\mathcal{U}}_{f}^{D}=\sup \underline{\mathcal{U}}_{f}^{D}=H_{f}^{D}
$$

where we have indicated with $\overline{\mathcal{U}}_{f}^{D}$ the collection of all $u \in \bar{S}(D)$ such that $\inf _{D} u>$ $-\infty$, and

$$
\liminf _{(x, t) \rightarrow\left(x_{0}, t_{0}\right)} u(x, t) \geq f\left(x_{0}, t_{0}\right), \forall\left(x_{0}, t_{0}\right) \in \partial_{p} D,
$$

and with $\underline{\mathcal{U}}_{f}^{D}$ the collection of all $u \in \underline{S}(D)$ for which $\sup _{D} u<\infty$, and

$$
\limsup _{(x, t) \rightarrow\left(x_{0}, t_{0}\right)} u(x, t) \leq f\left(x_{0}, t_{0}\right), \forall\left(x_{0}, t_{0}\right) \in \partial_{p} D .
$$

Lemma 2.4. Let $D \subset \mathbb{R}^{n+1}$ be a bounded open set, let $f \in C\left(\partial_{p} D\right)$ and let $u$ be the generalized Perron-Wiener-Brelot-Bauer solution to the problem in (2.11), i.e., $u=H_{f}^{D}$ where $H_{f}^{D}$ be defined as in (2.13). Then $u \in \Gamma^{2}(D)$.

Proof. This follows from Theorem 1.1 in [40].

In the following we are concerned with the issue of regular boundary points and we note, concerning the solvability of the Dirichlet problem for the operator $H$, that in [40] Uguzzoni developes what he refers to as a "cone criterion" for nondivergence equations modeled on Hörmander vector fields. This is a generalization of the well-known positive density condition of classical potential theory. We next describe his result in the setting of domains of the form $\Omega_{T}=\Omega \times(0, T)$, where $\Omega \subset \mathbb{R}^{n}$ is assumed to be a bounded domain. In [40] a bounded open set $\Omega$ is said to have outer positive $d$-density at $x_{0} \in \partial \Omega$ if there exist $r_{0}, \theta>0$ such that

$$
\left|B_{d}\left(x_{0}, r\right) \backslash \bar{\Omega}\right| \geq \theta\left|B_{d}\left(x_{0}, r\right)\right|, \text { for all } r \in\left(0, r_{0}\right) .
$$

Furthermore, if $r_{0}$ and $\theta$ can be chosen independently of $x_{0}$ then one says that $\Omega$ satisfies the outer positive $d$-density condition. The following lemma is a special case of [40, Theorem 4.1].

Lemma 2.5. Assume that $\Omega$ satisfies the outer positive d-density condition. Given $f \in C\left(\partial_{p} \Omega_{T}\right)$ and $g \in \Gamma^{\beta}\left(\Omega_{T}\right)$ for some $0<\beta \leq \sigma$, where $\sigma$ is the Hölder exponent in (1.4), there exists a unique solution $u \in \Gamma^{2+\beta}\left(\Omega_{T}\right) \cap C\left(\Omega_{T} \cup \partial_{p} \Omega_{T}\right)$ to the problem

$$
H u=g \text { in } \Omega_{T}, \quad u=f \text { on } \partial_{p} \Omega_{T} .
$$

In particular, $\Omega_{T}$ is $H$-regular for the Dirichlet problem (2.11). 


\subsection{NTA domains}

In this section we recall the notion of NTA domain with respect to the control distance $d(x, y)$ induced by the system $X=\left\{X_{1}, \ldots, X_{m}\right\}$. We recall that, when $d(x, y)=|x-y|$, the notion of NTA domain was introduced in [26] in connection with the study of the boundary behavior of nonnegative harmonic functions. The first study of NTA domains in a sub-Riemannian context was conducted in [7], where a large effort was devoted to the nontrivial question of the construction of examples. In that paper the relevant Fatou theory was also developed and, in particular, the doubling condition for harmonic measure, and the comparison theorem for quotients of nonnegative solutions of sub-Laplacians. Subsequently, in the papers $[10,11]$ the notion of NTA domain was combined with an intrinsic outer ball condition to obtain the complete solvability of the Dirichlet problem.

Given a bounded open set $\Omega \subset \mathbb{R}^{n}$, we recall that a ball $B_{d}(x, r)$ is $M$-nontangential in $\Omega$ (with respect to the metric $d$ ) if

$$
M^{-1} r<d\left(B_{d}(x, r), \partial \Omega\right)<M r .
$$

Furthermore, given $x, y \in \Omega$ a sequence of $M$-non-tangential balls in $\Omega$, $B_{d}\left(x_{1}, r_{1}\right), \ldots, B_{d}\left(x_{p}, r_{p}\right)$, is called a Harnack chain of length $p$ joining $x$ to $y$ if $x \in B_{d}\left(x_{1}, r_{1}\right), y \in B_{d}\left(x_{p}, r_{p}\right)$, and $B_{d}\left(x_{i}, r_{i}\right) \cap B_{d}\left(x_{i+1}, r_{i+1}\right) \neq \varnothing$ for $i \in\{1, \ldots, p-1\}$. We note that in this definition consecutive balls have comparable radii.

Definition 2.6. We say that a connected, bounded open set $\Omega \subset \mathbb{R}^{n}$ is a nontangentially accessible domain with respect to the system $X=\left\{X_{1}, \ldots, X_{m}\right\}$ (NTA domain, hereafter) if there exist $M, r_{0}>0$ for which:

(i) (Interior corkscrew condition) For any $x_{0} \in \partial \Omega$ and $r \leq r_{0}$ there exists $A_{r}\left(x_{0}\right) \in \Omega$ such that $M^{-1} r<d\left(A_{r}\left(x_{0}\right), x_{0}\right) \leq r$ and $\bar{d}\left(A_{r}\left(x_{0}\right), \partial \Omega\right)>$ $M^{-1} r$. (This implies that $B_{d}\left(A_{r}\left(x_{0}\right),(2 M)^{-1} r\right)$ is $(3 M)$-nontangential.)

(ii) (Exterior corkscrew condition) $\Omega^{c}=\mathbb{R}^{n} \backslash \Omega$ satisfies property (i).

(iii) (Harnack chain condition) There exists $C(M)>0$ such that for any $\epsilon>0$ and $x, y \in \Omega$ such that $d(x, \partial \Omega)>\epsilon, d(y, \partial \Omega)>\epsilon$, and $d(x, y)<C \epsilon$, there exists a Harnack chain joining $x$ to $y$ whose length depends on $C$ but not on $\epsilon$.

We observe that the Chow-Rashevski accessibility theorem implies that the metric space $\left(\mathbb{R}^{n}, d\right)$ be locally compact, see [23]. Furthermore, for any bounded set $\Omega \subset$ $\mathbb{R}^{n}$ there exists $R_{0}=R_{0}(\Omega)>0$ such that the closure of balls $B\left(x_{0}, R\right)$ with $x_{0} \in \Omega$ and $0<R<R_{0}$ are compact. We stress that metric balls of large radii fail to be compact in general, see [23]. In view of these observations, for a given NTA domain $\Omega \subset \mathbb{R}^{n}$ with constant $M$ and $r_{0}$ we will always assume, following [7], that the constant $r_{0}$ has been adjusted in such a way that the closure of balls $B\left(x_{0}, R\right)$, with $x_{0} \in \Omega$ and $0<R<r_{0}$, be compact.

We note the following lemma which will prove useful in the sequel and which follows directly from Lemma 2.5 and Definition 2.6. In its statement the number $\sigma$ denotes the Hölder exponent in (1.4). 
Lemma 2.7. Let $\Omega \subset \mathbb{R}^{n}$ be NTA domain, then there exist constants $C, R_{1}$, depending on the NTA parameters of $\Omega$, such that for every $y \in \partial \Omega$ and every $0<r<R_{1}$ one has,

$$
C\left|B_{d}(y, r)\right| \leq \min \left\{\left|\Omega \cap B_{d}(y, r)\right|,\left|\Omega^{c} \cap B_{d}(y, r)\right|\right\} \leq C^{-1}\left|B_{d}(y, r)\right| .
$$

In particular, every NTA domain has outer positive d-density and therefore, in view of Lemma 2.5, given $f \in C\left(\partial_{p} \Omega_{T}\right)$, there exists a unique solution $u \in \Gamma^{2+\sigma}\left(\Omega_{T}\right) \cap$ $C\left(\Omega_{T} \cup \partial_{p} \Omega_{T}\right)$ to the Dirichlet problem (2.11). In particular, $\Omega_{T}$ is H-regular.

Assume that $\Omega \subset \mathbb{R}^{n}$ is a non-tangentially accessible domain with respect to the system $X=\left\{X_{1}, \ldots, X_{m}\right\}$ and with parameters $M, r_{0}$. Let $T>0$ and define $\Omega_{T}=\Omega \times(0, T)$. Based on Definition 2.6, for every $\left(x_{0}, t_{0}\right) \in S_{T}, 0<r<r_{0}$, we introduce the following points of reference whenever

$$
\begin{aligned}
& A_{r}^{+}\left(x_{0}, t_{0}\right)=\left(A_{r}\left(x_{0}\right), t_{0}+2 r^{2}\right), \\
& A_{r}^{-}\left(x_{0}, t_{0}\right)=\left(A_{r}\left(x_{0}\right), t_{0}-2 r^{2}\right), \\
& A_{r}\left(x_{0}, t_{0}\right)=\left(A_{r}\left(x_{0}\right), t_{0}\right) .
\end{aligned}
$$

We note here that according to [30, Lemma 6.4], $\mathbb{R}^{n} \backslash B_{d}\left(x_{0}, R\right)$ satisfies condition (ii) in Definition 2.6, and thus it also satisfies the uniform outer positive $d$-density condition, and one can solve the Dirichlet problem there. Also note that the same is true of the intersection of two sets that satisfy condition (ii) in Definition 2.6. This is used to prove the following lemma ([30, Theorem 6.5]) which states that one can approximate any bounded open set with a set where one can solve the Dirichlet problem (2.11).

Lemma 2.8. Let $D \subset \mathbb{R}^{n}$ be a bounded open set. Then, for every $\delta>0$ there exists a set $D_{\delta}$ such that $\{x \in D: d(x, \partial D)>\delta\} \subset D_{\delta} \subset D$, and $D_{\delta}$ satisfies the uniform outer positive d-density condition.

To apply the Harnack inequality to the equation (1.1) in a cylinder $\Omega_{T}$, we will need to connect two points of $\Omega_{T}$ with a suitable Harnack chain of parabolic cylinders. We thus introduce the relevant geometric definition.

Definition 2.9. Let $\left(y_{1}, s_{1}\right),\left(y_{2}, s_{2}\right) \in \Omega_{T}$, with $s_{2}>s_{1}$. Suppose that $\left(s_{2}-\right.$ $\left.s_{1}\right)^{1 / 2} \geq \eta^{-1} d\left(y_{1}, y_{2}\right)$ for some $\eta>1$, and that $d\left(y_{1}, \partial \Omega\right)>\epsilon, d\left(y_{2}, \partial \Omega\right)>\epsilon$, $\left(T-s_{2}\right)>\epsilon^{2}, s_{1}>\epsilon^{2}$ and $d_{p}\left(\left(y_{1}, s_{1}\right),\left(y_{2}, s_{2}\right)\right)<c \epsilon$ for some $\epsilon>0$. We say that $\left\{C_{\hat{r}_{i}, \hat{\rho}_{i}}\left(\hat{y}_{i}, \hat{s}_{i}\right)\right\}_{i=1}^{\ell}$ is a parabolic Harnack chain of length $\ell$ connecting $\left(y_{1}, s_{1}\right)$ to $\left(y_{2}, s_{2}\right)$, if $\hat{r}_{i}, \hat{\rho}_{i}, \hat{y}_{i}, \hat{s}_{i}$ satisfy the following:

(i) $c(\eta)^{-1} \leq \frac{\hat{\rho}_{i}}{\hat{r}_{i}} \leq c(\eta)$ for $i=1,2, \ldots, \ell$,

(ii) $\hat{s}_{i+1}-\hat{s}_{i} \geq c(\eta)^{-1} \hat{r}_{i}^{2}$, for $i=1,2, \ldots, \ell-1$,

(iii) $B_{d}\left(\hat{y}_{i}, \hat{r}_{i}\right)$ is $M$-nontangential in $\Omega$ for $i=1,2, \ldots, \ell$,

(iv) $\left(y_{1}, s_{1}\right) \in C_{\hat{r}_{1}, \hat{\rho}_{1}}\left(\hat{y}_{1}, \hat{s}_{1}\right),\left(y_{2}, s_{2}\right) \in C_{\hat{r}_{\ell}, \hat{\rho}_{\ell}}\left(\hat{y}_{\ell}, \hat{s}_{\ell}\right)$,

(v) $C_{\hat{r}_{i+1}, \hat{\rho}_{i+1}}\left(\hat{y}_{i+1}, \hat{s}_{i+1}\right) \cap C_{\hat{r}_{i}, \hat{\rho}_{i}}\left(\hat{y}_{i}, \hat{s}_{i}\right) \neq \varnothing$ for $i=1,2, \ldots, \ell-1$. 
Lemma 2.10. Let $\Omega \subset \mathbb{R}^{n}$ be a NTA-domain. Given $T>0$ and $\left(y_{1}, s_{1}\right),\left(y_{2}, s_{2}\right) \in$ $\Omega_{T}$, suppose that $s_{2}>s_{1},\left(s_{2}-s_{1}\right)^{1 / 2} \geq \eta^{-1} d\left(y_{1}, y_{2}\right)$ for some $\eta>1$, that $d\left(y_{1}, \partial \Omega\right)>\epsilon, d\left(y_{2}, \partial \Omega\right)>\epsilon,\left(T-s_{2}\right)>\epsilon^{2}, s_{1}>\epsilon^{2}$ and that $d_{p}\left(\left(y_{1}, s_{1}\right),\left(y_{2}, s_{2}\right)\right)<$ $c \epsilon$ for some $\epsilon>0$. Then, there exists a parabolic Harnack chain $\left\{C_{\hat{r}_{i}, \hat{\rho}_{i}}\left(\hat{y}_{i}, \hat{s}_{i}\right)\right\}_{i=1}^{\ell}$, connecting $\left(y_{1}, s_{1}\right)$ to $\left(y_{2}, s_{2}\right)$ in the sense of Definition 2.9. Furthermore, the length $\ell$ of the chain can be chosen to depend only on $\eta$ and $c$, but not on $\epsilon$.

Proof. Since $\Omega$ is a NTA domain and since $d\left(y_{1}, \partial \Omega\right)>\epsilon, d\left(y_{2}, \partial \Omega\right)>\epsilon$,

$$
d\left(y_{1}, y_{2}\right) \leq d_{p}\left(\left(y_{1}, s_{1}\right),\left(y_{2}, s_{2}\right)\right)<c \epsilon,
$$

it follows that we can use Definition 2.6 to conclude the existence of a Harnack chain of length $\hat{\ell}=\hat{\ell}(c),\left\{B_{d}\left(\hat{y}_{i}, \hat{r}_{i}\right)\right\}_{i=1}^{\hat{\ell}}$, connecting $y_{1}$ and $y_{2}$. In the following we let $\beta$ be a degree of freedom to be fixed below. Using $\beta$ we define $\hat{\rho}_{i}=\beta \hat{r}_{i}$, we let $\hat{s}_{i}=s_{1}+\frac{1}{\beta} \sum_{j=1}^{i} \hat{r}_{j}^{2}$ for $i \in\{1, . ., \hat{\ell}\}$, and we consider the sequence of cylinders

$$
\left\{C_{\hat{r}_{i}, \hat{\rho}_{i}}\left(\hat{y}_{i}, \hat{s}_{i}\right)\right\}_{i=1}^{\hat{\ell}} \cdot
$$

If we now choose $\beta>1$, and if we assume that $\beta$ is chosen as a function of $\eta$, then (i), (ii), (iii), (v) and the first part of (iv) in Definition 2.9 are satisfied. In particular, it only remains to ensure that the second part of (iv) in Definition 2.9 is satisfied. To do this we first note that we can assume, without loss of generality, that $\hat{r}_{i} \leq d\left(y_{1}, y_{2}\right)$ for all $i \in\{1, \ldots, \hat{\ell}\}$. Hence, $\sum_{1}^{\hat{l}} \hat{r}_{i}^{2} \leq \hat{\ell} \cdot d\left(y_{1}, y_{2}\right)^{2}$. Furthermore, since $d\left(y_{1}, y_{2}\right)^{2} \leq \eta^{2}\left(s_{2}-s_{1}\right)$, we have

$$
\hat{s}_{\hat{\ell}}-s_{1}=\frac{1}{\beta} \sum_{1}^{\hat{\ell}} \hat{r}_{j}^{2} \leq \frac{\hat{\ell}}{\beta} d\left(y_{1}, y_{2}\right)^{2} \leq \frac{\hat{\ell}}{\beta} \eta^{2}\left(s_{2}-s_{1}\right) .
$$

We now let $\beta=\hat{\ell} \cdot \eta^{2}$ and we can conclude that $\hat{s}_{\hat{\ell}} \leq s_{2}$. If $\hat{s}_{\hat{\ell}}=s_{2}$ we are done. Otherwise, we only step up in time with cylinders $C_{j}=\left\{C_{\hat{r}_{\hat{\ell}}, \hat{r}_{\hat{\ell}}}\left(y_{2}, \hat{s}_{\hat{\ell}}+j \hat{r}_{\hat{\ell}}\right)\right\}$ until we reach $\left(y_{2}, s_{2}\right)$. The time that is left depends on $\eta$, and, in particular, we have that $s_{2}-\hat{s}_{\hat{\ell}} \leq c^{2} \epsilon^{2}$. Furthermore, since $\hat{r}_{\ell} \leq c \epsilon$, the number of steps we need to reach $\left(y_{2}, s_{2}\right)$ only depends on $c$. In particular, it is clear that the length of the entire parabolic Harnack chain only depends on $c$ and $\eta$.

Lemma 2.11. Let $u$ be a nonnegative solution to the equation $H u=0$ in $\Omega_{T}$. Furthermore, let $\left(y_{1}, s_{1}\right),\left(y_{2}, s_{2}\right) \in \Omega_{T}$, suppose that $s_{2}>s_{1},\left(s_{2}-s_{1}\right)^{1 / 2} \geq$ $\eta^{-1} d\left(y_{1}, y_{2}\right)$ for some $\eta>1$, that $d\left(y_{1}, \partial \Omega\right)>\epsilon, d\left(y_{2}, \partial \Omega\right)>\epsilon,\left(T-s_{2}\right)>\epsilon^{\overline{2}}$, $s_{1}>\epsilon^{2}$ and that $d_{p}\left(\left(y_{1}, s_{1}\right),\left(y_{2}, s_{2}\right)\right)<c \epsilon$ for some $\epsilon>0$. Then, there exists $a$ constant $\hat{c}=\hat{c}\left(H, \eta, c, r_{0}\right), 1 \leq \hat{c}<\infty$, such that

$$
u\left(y_{1}, s_{1}\right) \leq \hat{c} u\left(y_{2}, s_{2}\right) .
$$

Proof. To prove the lemma we simply use the parabolic Harnack chain from Lemma 2.10 and apply Theorem 2.2 in each cylinder. Note that the dependence of constant $\hat{c}$ on $r_{0}$ enters through the size parameter $R$ in the statement of Theorem 2.2. 


\section{Basic estimates}

The purpose of this section is to establish a number of basic technical estimates that will be used in the proof of Theorems 1.1-1.3. We mention that, using the notion of NTA domain and Lemma 2.11, several of the proofs previously established in the literature in the classical case $m=n$ and $\left\{X_{1}, \ldots, X_{m}\right\}=\left\{\partial_{x_{1}}, \ldots, \partial_{x_{n}}\right\}$ can be extended to our setting. As a consequence, wherever appropriate, we will either omit details or be brief. As previously, unless otherwise stated, $c$ will denote a positive constant $\geq 1$, not necessarily the same at each occurrence, depending only on $H$ and $M$. In general, $c\left(a_{1}, \ldots, a_{m}\right)$ denotes a positive constant $\geq 1$, which may depend only on $H, M$ and $a_{1}, \ldots, a_{m}$, and which is not necessarily the same at each occurrence. When we write $A \approx B$ we mean that $A / B$ is bounded from above and below by constants which, unless otherwise stated, only depend on $H, M$.

Lemma 3.1. Let $\left(x_{0}, t_{0}\right) \in S_{T}$ and

$$
r<\min \left\{r_{0} / 2, \sqrt{\left(T-t_{0}\right) / 4}, \sqrt{t_{0} / 4}\right\} .
$$

Let $u$ be a nonnegative solution to $H u=0$ in $\Omega_{T} \cap C_{2 r}\left(x_{0}, t_{0}\right)$ which vanishes continuously on $\Delta\left(x_{0}, t_{0}, 2 r\right)$. Then, there exist $c=c\left(H, M, r_{0}\right), 1 \leq c<\infty$, and $\gamma=\gamma(H, M)>0$, such that for every $(x, t) \in \Omega_{T} \cap C_{r}\left(x_{0}, t_{0}\right)$,

$$
u(x, t) d_{p}\left(x, t, S_{T}\right)^{\gamma} \leq c r^{\gamma} u\left(A_{r}^{+}\left(x_{0}, t_{0}\right)\right) .
$$

Proof. The proof of this lemma is based on Lemma 2.11. In particular, let $P_{0}=$ $(x, t) \in \Omega_{T} \cap C_{r}\left(x_{0}, t_{0}\right)$ and let $a=d_{p}\left(P_{0}, S_{T}\right)$. Note that, without loss of generality, we can assume that $a<r / c_{1}$ for some large $c_{1}$ since otherwise we are done immediately by a simple application of Lemma 2.11. Now, take $Q_{0} \in S_{T}$ such that $d_{p}\left(Q_{0}, P_{0}\right)=a$ and define $P_{i}=A_{2^{i} a}^{+}\left(Q_{0}\right)$ for all $i \geq 1$ such that $A_{2^{i} a}^{+}\left(Q_{0}\right)$ is well-defined. We intend to use Lemma 2.11 to prove that $u\left(P_{i}\right) \leq \operatorname{cu}\left(P_{i+1}\right)$ for some constant $c=c\left(H, M, r_{0}\right)$. In the following we write $P_{i}=\left(P_{i}^{x}, P_{i}^{t}\right)$, $Q_{0}=\left(Q_{0}^{x}, Q_{0}^{t}\right)$ to indicate the spatial and time coordinate of $P_{i}$ and $Q_{0}$ respectively. Then, for $i=0$ we have

$$
d\left(P_{0}^{x}, P_{1}^{x}\right) \leq d\left(P_{0}^{x}, Q_{0}^{x}\right)+d\left(Q_{0}^{x}, P_{1}^{x}\right) \leq a+2 a=\frac{3}{2 \sqrt{2}}\left(P_{0}^{t}-P_{1}^{t}\right)^{1 / 2} .
$$

Since $3 / 2 \sqrt{2}>1$, using Lemma 2.11 we can conclude that $u\left(P_{0}\right) \leq c u\left(P_{1}\right)$. To continue, for $i \geq 1$ we first note that

$$
P_{i+1}^{t}-P_{i}^{t}=2\left(2^{i+1} a\right)^{2}-2\left(2^{i} a\right)^{2}=3 \cdot 2^{2 i+1} a^{2} .
$$

Furthermore, we also have

$$
d\left(P_{i+1}^{x}, P_{i}^{x}\right) \leq d\left(P_{i+1}^{x}, Q_{0}^{x}\right)+d\left(Q_{0}^{x}, P_{i}^{x}\right) \leq 2^{i+1} a+2^{i} a=\sqrt{\frac{3}{2}}\left(P_{i+1}^{t}-P_{i}^{t}\right)^{1 / 2} .
$$


Let $\epsilon=2^{i} a / M$. Then $d\left(P_{i}^{x}, \partial \Omega\right)>\epsilon, d\left(P_{i+1}^{x}, \partial \Omega\right)>\epsilon$ and $d_{p}\left(P_{i+1}, P_{i}\right)=$ $\left(3 \cdot 2^{2 i} a^{2}+3 \cdot 2^{2 i+1} a^{2}\right)^{1 / 2}=\sqrt{15} M \cdot \epsilon$. Since $\sqrt{\frac{3}{2}}$ and $\sqrt{15} M$ are both independent of $i$ and since $\sqrt{\frac{3}{2}}>1$, we can again conclude, using Lemma 2.11, that $u\left(P_{i}\right) \leq$ $C u\left(P_{i+1}\right)$ for all $i>0$ such that $P_{i}$ and $P_{i+1}$ lie in $\Omega_{T} \cap C_{2 r}\left(x_{0}, t_{0}\right)$. In particular, to complete the proof it is now enough to consider the largest $k$ such that $2^{k} a \leq r$ and then iterate the above inequalities in a standard fashion. We omit further details.

Lemma 3.2. Let $\left(x_{0}, t_{0}\right) \in S_{T}$ and

$$
r<\min \left\{r_{0} / 2, \sqrt{\left(T-t_{0}\right) / 4}, \sqrt{t_{0} / 4}\right\} .
$$

Let $u$ be a nonnegative solution to $H u=0$ in $\Omega_{T} \cap C_{2 r}\left(x_{0}, t_{0}\right)$ vanishing continuously on $\Delta\left(x_{0}, t_{0}, 2 r\right)$. Then, there exist $c=c\left(H, M, r_{0}\right), 1 \leq c<\infty$, and $\gamma=\gamma\left(H, M, r_{0}\right)>0$, such that

$$
u\left(A_{r}^{-}\left(x_{0}, t_{0}\right)\right) \leq c\left(\frac{r}{d_{p}\left(x, t, \partial_{p} \Omega_{T}\right)}\right)^{\gamma} u(x, t),
$$

whenever $(x, t) \in \Omega_{T} \cap C_{r}\left(x_{0}, t_{0}\right)$.

Proof. To prove this lemma one can proceed similarly to the proof of Lemma 3.1.

Lemma 3.3. There exists a $\hat{K} \gg 1, \hat{K}=\hat{K}(H, M)$, such that the following is true whenever $\left(x_{0}, t_{0}\right) \in \mathbb{R}^{n+1}$ and $r<r_{0} /(2 \hat{K})$. Assume that $D$ is a domain in $\mathbb{R}^{n}$ such that $D \subset B_{d}\left(x_{0}, \hat{K} r\right)$ and assume that there exist $\hat{x}_{0} \in B_{d}\left(x_{0}, \hat{K} r\right)$ and $\rho>0$ such that $B_{d}\left(\hat{x}_{0}, 2 \rho\right) \subset B_{d}\left(x_{0}, r\right), B_{d}\left(\hat{x}_{0}, 2 \rho\right) \cap D=\varnothing$ and $M^{-1} r<\rho<r$. Let $u$ be $a$ function in $D \times\left(t_{0}-4 r^{2}, t_{0}\right)$ which satisfies $H u \geq 0$ in $D \times\left(t_{0}-4 r^{2}, t_{0}\right), u \leq 0$ on $\partial_{p}\left(D \times\left(t_{0}-4 r^{2}, t_{0}\right)\right) \backslash \partial_{p} C_{\hat{K} r, 2 r}^{-}\left(x_{0}, t_{0}\right)$ and $\sup _{D \times\left(t_{0}-4 r^{2}, t_{0}\right)} u>0$. Then, there exists a constant $\theta=\theta\left(H, M, r_{0}\right), 0<\theta<1$, such that

$$
\sup _{\left(D \times\left(t_{0}-4 r^{2}, t_{0}\right)\right) \cap C_{r}^{-}\left(x_{0}, t_{0}\right)} u \leq \theta \sup _{D \times\left(t_{0}-4 r^{2}, t_{0}\right)} u .
$$

Proof. Let $\hat{K} \gg 1$ be a constant to be fixed below. We let $\phi_{1} \in C_{0}^{\infty}\left(\mathbb{R}^{n}\right)$ be such that $0 \leq \phi_{1} \leq 1, \phi_{1} \equiv 1$ on $B_{d}\left(x_{0}, \hat{K} r+r\right) \backslash B_{d}\left(x_{0}, \hat{K} r-r\right), \phi_{1} \equiv 0$ on $B_{d}\left(x_{0}, \hat{K} r-2 r\right) \cup\left(\mathbb{R}^{n} \backslash B_{d}\left(x_{0}, \hat{K} r+2 r\right)\right)$. Similarly, we let $\phi_{2} \in C_{0}^{\infty}\left(\mathbb{R}^{n}\right)$ be such that $0 \leq \phi_{2} \leq 1, \phi_{2} \equiv 1$ on $B_{d}\left(x_{0}, \hat{K} r\right) \backslash B_{d}\left(\hat{x}_{0}, 2 \rho\right), \phi_{2} \equiv 0$ on $B_{d}\left(\hat{x}_{0}, \rho\right) \cup$ $\left(\mathbb{R}^{n} \backslash B_{d}\left(x_{0}, \hat{K} r+2 r\right)\right)$. Using $\phi_{1}$ and $\phi_{2}$ we define

$$
\begin{aligned}
& \Phi_{1}(\hat{x}, \hat{t})=\int_{\mathbb{R}^{n}} \Gamma\left(\hat{x}, \hat{t}, \xi, t_{0}-4 r^{2}\right) \phi_{1}(\xi) d \xi, \\
& \Phi_{2}(\hat{x}, \hat{t})=\int_{\mathbb{R}^{n}} \Gamma\left(\hat{x}, \hat{t}, \xi, t_{0}-4 r^{2}\right) \phi_{2}(\xi) d \xi,
\end{aligned}
$$


whenever $(\hat{x}, \hat{t}) \in \mathbb{R}^{n+1}, \hat{t} \geq t_{0}-4 r^{2}$. To preceed we first prove that there exist a constant $c$ such that

$$
1 \leq c \Phi_{1}(\hat{x}, \hat{t}) \text { for }(\hat{x}, \hat{t}) \in \partial_{p}\left(C_{\hat{K} r, 2 r}^{-}\left(x_{0}, t_{0}\right) \cap\left\{(x, t): t_{0}-4 r^{2}<t<t_{0}\right\}\right) .
$$

To establish this, let $(\hat{x}, \hat{t})$ be as in (3.2), and for simplicity assume that $t_{0}-4 r^{2}=0$. Then, using Lemma 2.1 and (2.2) we see that

$$
\begin{aligned}
\Phi_{1}(\hat{x}, \hat{t}) & \geq \int_{B_{d}(\hat{x}, \sqrt{\hat{t}} / 2)} \Gamma(\hat{x}, \hat{t}, \xi, 0) \phi_{1}(\xi) d \xi \\
& \geq \int_{B_{d}(\hat{x}, \sqrt{\hat{t}} / 2)} c^{-1}|B(\hat{x}, \sqrt{\hat{t}})|^{-1} e^{-C d(\hat{x}, \xi)^{2} / \hat{t}} d \xi \\
& =e^{-C \hat{t} / 4 \hat{t}} \int_{B_{d}(\hat{x}, \sqrt{\hat{t}} / 2)} c^{-1}|B(\hat{x}, \sqrt{\hat{t}})|^{-1} e^{-C\left(4 d(\hat{x}, \xi)^{2}-\hat{t}\right) / 4 \hat{t}} d \xi \\
& \geq e^{-C / 4} c^{-1}|B(\hat{x}, \sqrt{\hat{t}})|^{-1} \int_{B_{d}(\hat{x}, \sqrt{\hat{t}} / 2)} d \xi \geq e^{-C / 4} c^{-1} \hat{C}^{-1} .
\end{aligned}
$$

We conclude that (3.2) holds provided that we choose $c \leq e^{-C / 4} \hat{C}^{-1}$. Now, let

$$
M=\sup _{D \times\left(t_{0}-4 r^{2}, t_{0}\right)} u .
$$

Using (3.2) and the maximum principle on $D \times\left(t_{0}-4 r^{2}, t_{0}\right)$ we thus see that the estimate

$$
u(\hat{x}, \hat{t}) \leq c M \Phi_{1}(\hat{x}, \hat{t})+M \Phi_{2}(\hat{x}, \hat{t})
$$

holds in $D \times\left(t_{0}-4 r^{2}, t_{0}\right)$, and thus in particular in $\left(D \times\left(t_{0}-4 r^{2}, t_{0}\right)\right) \cap C_{r}^{-}\left(x_{0}, t_{0}\right)$. Further, if $(\hat{x}, \hat{t}) \in\left(D \times\left(t_{0}-4 r^{2}, t_{0}\right)\right) \cap C_{r}^{-}\left(x_{0}, t_{0}\right)$, then

$$
\begin{aligned}
& \Phi_{1}(\hat{x}, \hat{t}) \leq \int_{B_{d}\left(x_{0}, \hat{K} r+r\right) \backslash B_{d}\left(x_{0}, \hat{K} r-r\right)}|B(\hat{x}, \sqrt{\hat{t}})|^{-1} e^{-C^{-1} d(\hat{x}, \xi)^{2} / \hat{t}} d \xi \\
& \leq \int_{B_{d}\left(x_{0}, \hat{K} r+r\right) \backslash B_{d}\left(x_{0}, \hat{K} r-r\right)}|B(\hat{x}, r)|^{-1} e^{-c^{-1} d(\hat{x}, \xi)^{2} / r^{2}} d \xi \\
& \leq c e^{-c^{-1} \hat{K}^{2}}\left|B_{d}\left(x_{0}, \hat{K} r+r\right) \backslash B_{d}\left(x_{0}, \hat{K} r-r\right)\right||B(\hat{x}, r)|^{-1} \\
& \leq c e^{-c^{-1} \hat{K}^{2}}\left|B_{d}\left(x_{0}, \hat{K} r+r\right)\right||B(\hat{x}, r)|^{-1} \text {. }
\end{aligned}
$$

Iterating (2.2) and using that $r<r_{0} /(2 \hat{K})$ we see that

$$
c e^{-c^{-1} \hat{K}^{2}}\left|B_{d}\left(x_{0}, \hat{K} r+r\right)\right||B(\hat{x}, r)|^{-1} \leq c e^{-c^{-1} \hat{K}^{2}} \hat{K}^{\eta}
$$

for some integer $\eta \gg 1$ which is independent of $\hat{K}, x_{0}, \hat{x}$ and $r$. In particular

$$
\Phi_{1}(\hat{x}, \hat{t}) \leq c e^{-c^{-1} \hat{K}^{2}} \hat{K}^{\eta} .
$$


To estimate $\Phi_{2}(\hat{x}, \hat{t})$ we note that

$$
\begin{aligned}
& \Phi_{2}(\hat{x}, \hat{t})=1-\hat{\Phi}_{2}(\hat{x}, \hat{t}), \text { where } \\
& \hat{\Phi}_{2}(\hat{x}, \hat{t})=\int_{\mathbb{R}^{n}} \Gamma\left(\hat{x}, \hat{t}, \xi, t_{0}-4 r^{2}\right)\left(1-\phi_{2}(\xi)\right) d \xi
\end{aligned}
$$

and by construction,

$$
\hat{\Phi}_{2}(\hat{x}, \hat{t}) \geq \int_{B_{d}(\hat{x}, \rho)} \Gamma\left(\hat{x}, \hat{t}, \xi, t_{0}-4 r^{2}\right) d \xi .
$$

As before we then prove that

$$
\hat{\Phi}_{2}(\hat{x}, \hat{t}) \geq c^{-1}
$$

and actually, for $\varepsilon$ small enough,

$$
\hat{\Phi}_{2}\left(\hat{x}_{0}, t_{0}-4 r^{2}+\varepsilon^{2} \rho^{2}\right) \geq c^{-1} .
$$

Hence, by using the Harnack inequality we can conclude that $\Phi_{2}(\hat{x}, \hat{t})=1-$ $\hat{\Phi}_{2}(\hat{x}, \hat{t}) \leq\left(1-c^{-1}\right)$, whenever $(\hat{x}, \hat{t}) \in\left(D \times\left(t_{0}-4 r^{2}, t_{0}\right)\right) \cap C_{r}^{-}\left(x_{0}, t_{0}\right)$ for some $c=c\left(H, M, r_{0}\right)>1$. In particular, for every $(\hat{x}, \hat{t}) \in\left(D \times\left(t_{0}-4 r^{2}, t_{0}\right)\right) \cap$ $C_{r}^{-}\left(x_{0}, t_{0}\right)$, we have

$$
u(\hat{x}, \hat{t}) \leq c M \Phi_{1}(\hat{x}, \hat{t})+M \Phi_{2}(\hat{x}, \hat{t}) \leq M\left(c e^{-c^{-1} \hat{K}^{2}} \hat{K}^{\eta}+\left(1-\hat{c}^{-1}\right)\right),
$$

for some $\hat{c}=\hat{c}\left(H, M, r_{0}\right)$. Given $\hat{c}$, we choose $\hat{K}$ so that $c e^{-c^{-1} \hat{K}^{2}} \hat{K}^{\eta} \leq \hat{c}^{-1} / 2$, and we let $\theta=\left(1-\hat{c}^{-1} / 2\right)<1$. Then, the following inequality holds

$$
u(\hat{x}, \hat{t}) \leq \theta M
$$

with $M$ as in (3.3). This establishes (3.1), thus completing the proof.

We will also need a few variations on the theme of Lemma 3.3.

Corollary 3.4. There exists a $\hat{K} \gg 1, \hat{K}=\hat{K}\left(H, M, r_{0}\right)$, such that the following is true whenever $\left(x_{0}, t_{0}\right) \in S_{T}$ and

$$
r<\min \left\{r_{0} /(2 \hat{K}), \sqrt{\left(T-t_{0}\right) / 4}, \sqrt{t_{0} / 4}\right\} .
$$

Let $u$ be a nonnegative solution to $H u=0$ in $\Omega_{T} \cap C_{\hat{K} r, 2 r}^{-}\left(x_{0}, t_{0}\right)$ vanishing continuously on $S_{T} \cap C_{\hat{K} r, 2 r}^{-}\left(x_{0}, t_{0}\right)$. Then, there exists a constant $\theta=\theta(H, M)$, $0<\theta<1$, such that

$$
\sup _{\Omega_{T} \cap C_{r}^{-}\left(x_{0}, t_{0}\right)} u \leq \theta \underset{\Omega_{T} \cap C_{\hat{K} r, 2 r}^{-}\left(x_{0}, t_{0}\right)}{\sup } u .
$$


Proof. This is an obvious consequence of the NTA character of $\Omega$ and of Lemma 3.3. We omit further details.

Lemma 3.5. There exists a $\hat{K} \gg 1, \hat{K}=\hat{K}\left(H, M, r_{0}\right)$, such that the following is true whenever $\left(x_{0}, t_{0}\right) \in S_{T}$ and

$$
r<\min \left\{r_{0} /(2 \hat{K}), \sqrt{\left(T-t_{0}\right) /(4 \hat{K})^{2}}, \sqrt{t_{0} /(4 \hat{K})^{2}}\right\} .
$$

Let $u$ be a solution to $H u=0$ in $\Omega_{T} \cap C_{\hat{K} r, 2 r}^{-}\left(x_{0}, t_{0}\right)$ which vanishes continuously on $S_{T} \cap C_{\hat{K} r, 2 r}^{-}\left(x_{0}, t_{0}\right)$. Then, there exists a constant $\theta=\theta(H, M), 0<\theta<1$, such that

$$
\sup _{\Omega_{T} \cap C_{r}^{-}\left(x_{0}, t_{0}\right)} u^{ \pm} \leq \theta \quad \sup _{\Omega_{T} \cap C_{\hat{K} r, 2 r}^{-}} u_{0} u^{ \pm},
$$

where $u^{+}(x, t)=\max \{0, u(x, t)\}, u^{-}(x, t)=-\min \{0, u(x, t)\}$.

Proof. We first prove Lemma 3.5 for $u^{+}$. In fact, in this case the argument is essentially the same as that in the proof of Lemma 3.3. In particular, if we let

$$
\left.M^{+}=\sup _{\Omega_{T} \cap C_{\hat{K} r, 2 r}^{-}} u_{0}, t_{0}\right)
$$

then we see that (3.4) still holds but with $M$ replaced by $M^{+}$. Furthermore, repeating the argument in (3.3)-(3.5), we see that

$$
u(\hat{x}, \hat{t}) \leq \theta M^{+}
$$

whenever $(\hat{x}, \hat{t}) \in \Omega_{T} \cap C_{r}^{-}\left(x_{0}, t_{0}\right)$. Obviously this completes the proof of Lemma 3.5 for $u^{+}$. Concerning the same estimate for $u^{-}$we see, by analogy, that

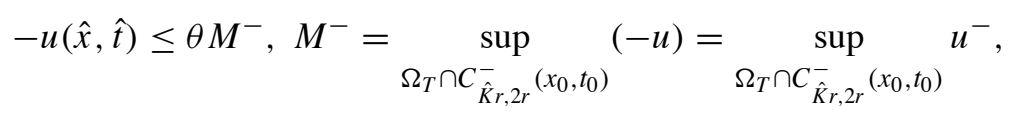

whenever $(\hat{x}, \hat{t}) \in \Omega_{T} \cap C_{r}^{-}\left(x_{0}, t_{0}\right)$ and from (3.6) we deduce Lemma 3.5 for $u^{-}$. This completes the proof of the lemma.

Lemma 3.6. Let $\left(x_{0}, t_{0}\right) \in S_{T}$ and let $r<\min \left\{r_{0} / 2, \sqrt{\left(T-t_{0}\right) / 4}, \sqrt{t_{0} / 4}\right\}$. Let $u$ be a nonnegative solution to $H u=0$ in $\Omega_{T} \cap C_{2 r}\left(x_{0}, t_{0}\right)$ which vanishes continuously on $\Delta\left(x_{0}, t_{0}, 2 r\right)$. Then, there exist a constant $c=c\left(H, M, r_{0}\right), 1 \leq c<\infty$, and $\alpha=\alpha(H, M) \in(0,1)$, such that

$$
u(x, t) \leq c\left(\frac{d_{p}\left(x, t, x_{0}, t_{0}\right)}{r}\right)^{\alpha} \sup _{\Omega_{T} \cap C_{2 r}\left(x_{0}, t_{0}\right)} u
$$

whenever $(x, t) \in \Omega_{T} \cap C_{r / c}\left(x_{0}, t_{0}\right)$. 
Proof. This lemma is a simple consequence of Corollary 3.4.

Lemma 3.7. Let $\left(x_{0}, t_{0}\right) \in S_{T}$ and let $r<\min \left\{r_{0} / 2, \sqrt{\left(T-t_{0}\right) / 4}, \sqrt{t_{0} / 4}\right\}$. Let $u$ be a nonnegative solution to $H u=0$ in $\Omega_{T} \cap C_{2 r}\left(x_{0}, t_{0}\right)$ vanishing continuously on $\Delta\left(x_{0}, t_{0}, 2 r\right)$. Then, there exists a constant $c=c\left(H, M, r_{0}\right), 1 \leq c<\infty$, such that

$$
u(x, t) \leq c u\left(A_{r}^{+}\left(x_{0}, t_{0}\right)\right)
$$

whenever $(x, t) \in \Omega_{T} \cap C_{r / c}\left(x_{0}, t_{0}\right)$.

Proof. This lemma is a consequence of Lemma 3.6, the Harnack inequality and a classical argument developed in [6] and [38].

Remark 3.8. Note that if $u$ is a nonnegative solution to $H u=0$ in all of $\Omega_{T}$ then Lemma 3.7 can be improved in the following way. Let $\left(x_{0}, t_{0}\right) \in S_{T}$ and $r$ be as in the statement of Lemma 3.7. Let $u$ be a nonnegative solution to $H u=0$ in $\Omega_{T}$ vanishing continuously on $\Delta\left(x_{0}, t_{0}, 2 r\right)$. Then, there exists a constant $c=$ $c\left(H, M, r_{0}\right), 1 \leq c<\infty$, such that

$$
u(x, t) \leq c u\left(A_{r}^{+}\left(x_{0}, t_{0}\right)\right)
$$

whenever $(x, t) \in \Omega_{T} \cap C_{r}\left(x_{0}, t_{0}\right)$. In fact, the restriction $(x, t) \in \Omega_{T} \cap C_{r / c}\left(x_{0}, t_{0}\right)$ in Lemma 3.7 is simply a result of the fact that we in Lemma 3.7 are only assuming that $u$ is a nonnegative solution in $\Omega_{T} \cap C_{2 r}\left(x_{0}, t_{0}\right)$.

Lemma 3.9. Let $u$ be a nonnegative solution to $H u=0$ in $\Omega_{T}$ which vanishes continuously on $S_{T}$. Let $0<\delta \ll \sqrt{T}$ be given. Then, there exists a constant $c=c\left(H, M, \operatorname{diam}(\Omega), T, \delta, r_{0}\right), 1 \leq c<\infty$, such that

$$
\sup _{(x, t) \in \Omega^{\delta} \times\left(\delta^{2}, T\right)} u(x, t) \leq c \inf _{(x, t) \in \Omega^{\delta} \times\left(\delta^{2}, T\right)} u(x, t) .
$$

Proof. To prove this we can proceed, using the lemmas given above, exactly as in the proof of [34, Lemma 2.7].

Lemma 3.10. Let $K \gg 1$ be given, let $\left(x_{0}, t_{0}\right) \in S_{T}$ and assume that $r<$ $\min \left\{r_{0} /(8 K), \sqrt{\left(T-t_{0}\right) / 64}, \sqrt{t_{0} / 64}\right\}$. Let $u$ be a nonnegative solution to the equation $H u=0$ in $\Omega_{T}$ vanishing continuously on $S_{T}$. Let $\gamma=\gamma(H, M) \in(0,1)$ be as in Lemma 3.1 and Lemma 3.2. Assume that

$$
\sup _{\Omega_{T} \cap C_{2 K r, 2 r}^{-}\left(x_{0}, t_{0}\right)} u \geq(2 K)^{-\gamma} \sup _{\Omega_{T} \cap C_{4 K r, 8 r}^{-}\left(x_{0}, t_{0}\right)} u .
$$

Then, provided $K=K(H, M)$ is chosen large enough, there exists $c=c\left(H, M, r_{0}\right) \geq$ 1, such that

$$
\sup _{\Omega_{T} \cap C_{4 K r}^{-}\left(x_{0}, t_{0}\right) \cap\left\{(x, t): t=t_{0}-64 r^{2}\right\}} u \geq c^{-1} \sup _{\Omega_{T} \cap C_{2 K r, 2 r}^{-}\left(x_{0}, t_{0}\right)} u .
$$


Proof. The proof of this lemma is similar to that of Lemma 3.4. In particular, we let $\phi_{1} \in C_{0}^{\infty}\left(\mathbb{R}^{n}\right)$ be such that $0 \leq \phi_{1} \leq 1, \phi_{1} \equiv 1$ on $B_{d}\left(x_{0}, 4 K r+2 r\right) \backslash B_{d}\left(x_{0}, 4 K r-\right.$ $2 r), \phi_{1} \equiv 0$ on $B_{d}\left(x_{0}, 4 K r-4 r\right) \cup\left(\mathbb{R}^{n} \backslash B_{d}\left(x_{0}, 4 K r+4 r\right)\right)$. Since $\Omega$ is NTA we see that there exist $\hat{x}_{0}$ and $\rho>0$ such that $r / M<4 \rho<r$ and such that $B\left(\hat{x}_{0}, 2 \rho\right) \subset$ $\left(\mathbb{R}^{n} \backslash \Omega\right) \cap B\left(x_{0}, r\right)$. Based on this we let $\phi_{2} \in C_{0}^{\infty}\left(\mathbb{R}^{n}\right)$ be such that $0 \leq \phi_{2} \leq 1$, $\phi_{2} \equiv 1$ on $B_{d}\left(x_{0}, 4 K r\right) \backslash B\left(\hat{x}_{0}, 2 \rho\right), \phi_{2} \equiv 0$ on $\left(\mathbb{R}^{n} \backslash B_{d}\left(x_{0}, 4 K r+4 r\right)\right) \cup B\left(\hat{x}_{0}, \rho\right)$. Using $\phi_{1}$ and $\phi_{2}$ we define

$$
\begin{aligned}
& \Phi_{1}(\hat{x}, \hat{t})=\int_{\mathbb{R}^{n}} \Gamma\left(\hat{x}, \hat{t}, \xi, t_{0}-64 r^{2}\right) \phi_{1}(\xi) d \xi, \\
& \Phi_{2}(\hat{x}, \hat{t})=\int_{\mathbb{R}^{n}} \Gamma\left(\hat{x}, \hat{t}, \xi, t_{0}-64 r^{2}\right) \phi_{2}(\xi) d \xi,
\end{aligned}
$$

whenever $(\hat{x}, \hat{t}) \in \mathbb{R}^{n+1}, \hat{t} \geq t_{0}-64 r^{2}$. Let $\Gamma_{1}=\Omega_{T} \cap C_{4 K r}^{-}\left(x_{0}, t_{0}\right) \cap\{(x, t): t=$ $\left.t_{0}-64 r^{2}\right\}, \Gamma_{2}=\partial_{p}\left(\Omega_{T} \cap C_{4 K r, 8 r}^{-}\left(x_{0}, t_{0}\right)\right) \backslash \Gamma_{1} \backslash S_{T}$. In the following we let

$$
M=\sup _{\Omega_{T} \cap C_{4 K r, 8 r}^{-}\left(x_{0}, t_{0}\right)} u, \hat{M}=\sup _{\Omega_{T} \cap C_{4 K r}^{-}\left(x_{0}, t_{0}\right) \cap\left\{(x, t): t=t_{0}-64 r^{2}\right\}} u .
$$

Then, by arguing as in the proof of Lemma 3.4, we first see that there exists $c$ such that

$$
1 \leq c \Phi_{1}(\hat{x}, \hat{t}) \text { for }(\hat{x}, \hat{t}) \in \partial_{p}\left(C_{4 K r, 8 r}^{-}\left(x_{0}, t_{0}\right)\right) \cap\left\{(x, t): t_{0}-64 r^{2}<t<t_{0}\right\},
$$

and then, by the maximum principle we see, that

$$
u(\hat{x}, \hat{t}) \leq c M \Phi_{1}(\hat{x}, \hat{t})+\hat{M} \Phi_{2}(\hat{x}, \hat{t})
$$

for $(\hat{x}, \hat{t}) \in \Omega_{T} \cap C_{2 K r, 2 r}^{-}\left(x_{0}, t_{0}\right)$. As in the proof of Lemma 3.4 we can then deduce that

$$
u(\hat{x}, \hat{t}) \leq c M e^{-c^{-1} K^{2}} K^{\eta}+\hat{M} \Phi_{2}(\hat{x}, \hat{t}),
$$

for $(\hat{x}, \hat{t}) \in \Omega_{T} \cap C_{2 K r, 2 r}^{-}\left(x_{0}, t_{0}\right)$. Next, using the assumption stated in the lemma we see that

$$
(2 K)^{-\gamma} M \leq c M e^{-c^{-1} K^{2}} K^{\eta}+\hat{M} \sup _{\Omega_{T} \cap C_{2 K r, 2 r}^{-}\left(x_{0}, t_{0}\right)} \Phi_{2}(\hat{x}, \hat{t}) .
$$

Hence, assuming that $K$ is so large that $(2 K)^{-\gamma}>c e^{-c^{-1} K^{2}} K^{\eta}$, we have that

$$
\left((2 K)^{-\gamma}-e^{-c^{-1} K^{2}} K^{\eta}\right) M \leq \hat{M} \sup _{\Omega_{T} \cap C_{2 K r, 2 r}^{-}\left(x_{0}, t_{0}\right)} \Phi_{2}(\hat{x}, \hat{t}) \leq \hat{M}
$$

In particular, we can conclude, for $K=K(H, M)$ large enough, that

$$
\frac{1}{2}(2 K)^{-\gamma} \sup _{\Omega_{T} \cap C_{2 K r, 2 r}^{-}\left(x_{0}, t_{0}\right)} u \leq \frac{1}{2}(2 K)^{-\gamma} M \leq \hat{M} .
$$

This completes the proof. 
Lemma 3.11. Let $\hat{K}$ be as in the statement of Lemma 3.3, let $K \gg \hat{K}$ be a constant to be suitably chosen, $\left(x_{0}, t_{0}\right) \in S_{T}$ and assume

$$
r<\min \left\{r_{0} /(2 K \hat{K}), \sqrt{\left(T-t_{0}\right) /\left(4 K^{2}\right)}, \sqrt{t_{0} /\left(4 K^{2}\right)}\right\} .
$$

Let $u$ be a solution to $H u=0$ in $\left(\Omega_{T} \backslash \Omega_{T}^{r}\right) \cap C_{K r}^{-}\left(x_{0}, t_{0}\right)$ which is continuous on the closure of $\left(\Omega_{T} \backslash \Omega_{T}^{r}\right) \cap C_{K r}^{-}\left(x_{0}, t_{0}\right)$. Moreover, assume that

(i) $u(x, t) \leq 1$ whenever $(x, t) \in\left(\Omega_{T} \backslash \Omega_{T}^{r}\right) \cap C_{K r}^{-}\left(x_{0}, t_{0}\right)$,

(ii) $u(x, t) \leq 0$ whenever $(x, t) \in\left[\left(\partial \Omega \cup \partial \Omega^{r}\right) \times\left(t_{0}-(K r)^{2}, t_{0}\right)\right] \cap C_{K r}^{-}\left(x_{0}, t_{0}\right)$.

Then, there exists a constant $c=c\left(H, M, r_{0}\right), 1 \leq c<\infty$, such that

$$
u(x, t) \leq e^{-c K}
$$

whenever $(x, t) \in\left(\Omega_{T} \backslash \Omega_{T}^{r}\right) \cap C_{\hat{K} r}^{-}\left(x_{0}, t_{0}\right)$.

Proof. In the following we consider odd integers $2 j+1$ where $j \in[0,(K / \hat{K}-$ 1)/2]. For each such $j$ we define a point $\left(\hat{X}_{j}, \hat{t}_{j}\right) \in\left(\Omega_{T} \backslash \Omega_{T}^{r}\right) \cap C_{(2 j+1) \hat{K} r}^{-}\left(x_{0}, t_{0}\right)$ through the relation

$$
\sup _{\left(\Omega_{T} \backslash \Omega_{T}^{r}\right) \cap C_{(2 j+1) \hat{K}_{r}}^{-}\left(x_{0}, t_{0}\right)} u=u\left(\hat{X}_{j}, \hat{t}_{j}\right) .
$$

We then note, using the maximum principle, that $\left(\hat{X}_{j}, \hat{t}_{j}\right) \in \partial_{p}\left[\left(\Omega_{T} \backslash \Omega_{T}^{r}\right) \cap\right.$ $\left.C_{(2 j+1) \hat{K} r}^{-}\left(x_{0}, t_{0}\right)\right]$. By construction we also see that there exists $\left(\tilde{X}_{j}, \hat{t}_{j}\right) \in \partial \Omega \times$ $\left[t_{0}-((2 j+1) \hat{K} r)^{2}, t_{0}\right)$ such that $d_{p}\left(\tilde{X}_{j}, \hat{t}_{j}, \hat{X}_{j}, \hat{t}_{j}\right)=d\left(\tilde{X}_{j}, \hat{X}_{j}\right) \leq r$. In particular, $\left(\hat{X}_{j}, \hat{t}_{j}\right)$ is in the closure of $C_{r}^{-}\left(\tilde{X}_{j}, \hat{t}_{j}\right)$. We next note that

$$
C_{\hat{K} r, 2 r}^{-}\left(\tilde{X}_{j}, \hat{t}_{j}\right) \cap\left[\left(\Omega_{T} \backslash \Omega_{T}^{r}\right) \cap C_{K r}^{-}\left(x_{0}, t_{0}\right)\right] \subset\left(\Omega_{T} \backslash \Omega_{T}^{r}\right) \cap C_{(2 j+3) \hat{K} r}^{-}\left(x_{0}, t_{0}\right) .
$$

Let $D$ be defined through the relation $D \times\left(\hat{t}_{j}-4 r^{2}, \hat{t}_{j}\right)=C_{\hat{K} r, 2 r}^{-}\left(\tilde{X}_{j}, \hat{t}_{j}\right) \cap$ $\left[\left(\Omega_{T} \backslash \Omega_{T}^{r}\right) \cap C_{K r}^{-}\left(x_{0}, t_{0}\right)\right]$. Then, applying Lemma 3.3 we see that there exists $\theta=\theta(H, M), 0<\theta<1$, such that

$$
\sup _{\left(D \times\left(\hat{t}_{j}-4 r^{2}, \hat{t}_{j}\right)\right) \cap C_{r}^{-}\left(\tilde{X}_{j}, \hat{t}_{j}\right)} u \leq \theta \sup _{D \times\left(\hat{t}_{j}-4 r^{2}, \hat{t}_{j}\right)} u .
$$

In particular, since $\left(\hat{X}_{j}, \hat{t}_{j}\right)$ is in the closure of the set $\left(D \times\left(\hat{t}_{j}-4 r^{2}, \hat{t}_{j}\right)\right) \cap$ $C_{r}^{-}\left(\tilde{X}_{j}, \hat{t}_{j}\right)$ we can use continuity of $u,(3.10)$ and (3.9) to conclude that

$$
\begin{aligned}
u\left(\hat{X}_{j}, \hat{t}_{j}\right) & \leq \theta \sup _{D \times\left(\hat{t}_{j}-4 r^{2}, \hat{t}_{j}\right)} u \\
& \leq \theta \sup _{\left(\Omega_{T} \backslash \Omega_{T}^{r}\right) \cap C_{(2 j+3) \hat{K}_{r}}^{-}\left(x_{0}, t_{0}\right)} u=\theta u\left(\hat{X}_{j+1}, \hat{t}_{j+1}\right) .
\end{aligned}
$$


Let $j_{0}$ be the largest positive integer such that $\left(2 j_{0}+3\right) \hat{K} \leq K$. Then, by iteration we see that

$$
\sup _{\left(\Omega_{T} \backslash \Omega_{T}^{r}\right) \cap C_{\hat{K} r}^{-}\left(x_{0}, t_{0}\right)} u=u\left(\hat{X}_{1}, \hat{t}_{1}\right) \leq \theta^{j_{0}} u\left(\hat{X}_{j_{0}+1}, \hat{t}_{j_{0}+1}\right) \leq \theta^{j_{0}}
$$

where we, at the last step, has used that $u(x, t) \leq 1$. Hence

$$
\sup _{\left(\Omega_{T} \backslash \Omega_{T}^{r}\right) \cap C_{\hat{K} r}^{-}\left(x_{0}, t_{0}\right)} u \leq \theta^{j_{0}} .
$$

Obviously (3.13) implies the statement in Lemma 3.11 and the proof is complete.

Lemma 3.12. Let $\hat{K}$ be as in the statement of Lemma 3.3, let $K \gg \hat{K}$ be given, $\left(x_{0}, t_{0}\right) \in S_{T}$ and assume that

$$
r<\min \left\{r_{0} /(2 K), \sqrt{\left(T-t_{0}\right) /\left(4 K^{2}\right)}, \sqrt{t_{0} /\left(4 K^{2}\right)}\right\}
$$

Let $u$ and $v$ be two solutions to $H u=0$ in $\left(\Omega_{T} \backslash \Omega_{T}^{r}\right) \cap C_{K r}^{-}\left(x_{0}, t_{0}\right)$. Moreover, assume that

(i) $u(x, t) \geq 0, v(x, t) \leq 1$ whenever $(x, t) \in\left(\Omega_{T} \backslash \Omega_{T}^{r}\right) \cap C_{K r}^{-}\left(x_{0}, t_{0}\right)$,

(ii) $u(x, t) \geq 1$ whenever $(x, t) \in\left[\partial \Omega^{r} \times\left(t_{0}-(K r)^{2}, t_{0}\right)\right] \cap C_{K r}^{-}\left(x_{0}, t_{0}\right)$,

(iii) $v(x, t) \leq 0$ whenever $(x, t) \in\left[\left(\partial \Omega \cup \partial \Omega^{r}\right) \times\left(t_{0}-(K r)^{2}, t_{0}\right)\right] \cap C_{K r}^{-}\left(x_{0}, t_{0}\right)$.

Then, for any $(x, t) \in \Omega_{T} \cap C_{r}^{-}\left(x_{0}, t_{0}\right)$ one has

$$
v(x, t) \leq u(x, t)
$$

provided $K=K(H, M)$ is chosen large enough.

Proof. To start the proof of Lemma 3.12 we claim that if $u$ as in the statement of the lemma, then

$$
u(x, t) \geq 2 \epsilon\left(\frac{d_{p}\left(x, t, S_{T}\right)}{r}\right)^{\eta} \text { whenever }(x, t) \in \Omega_{T} \cap C_{r}^{-}\left(x_{0}, t_{0}\right),
$$

where $\epsilon$ and $\eta$ are positive constants depending only on $H, M$. However, we postpone the proof of this claim until the end. We thus establish the lemma assuming (3.14). To do this we first note that (3.14) implies that

$$
u(x, t) \geq 2 \epsilon K^{-\eta} \text { whenever }(x, t) \in \Omega_{T}^{r / K} \cap C_{r}^{-}\left(x_{0}, t_{0}\right) .
$$


Furthermore, since $v$ satisfies the assumptions stated in Lemma 3.11, from this result we see that

$$
v(x, t) \leq e^{-c K} \leq \epsilon K^{-\eta} \text { whenever }(x, t) \in \Omega_{T} \cap C_{r}^{-}\left(x_{0}, t_{0}\right),
$$

provided $K=K(H, M)$ is large enough. In particular,

$$
v(x, t) \leq \epsilon K^{-\eta} \leq u(x, t) \text { whenever }(x, t) \in \Omega_{T}^{r / K} \cap C_{r}^{-}\left(x_{0}, t_{0}\right) .
$$

We now define for $(x, t) \in\left(\Omega_{T} \backslash \Omega_{T}^{r / K}\right) \cap C_{r}^{-}\left(x_{0}, t_{0}\right)$,

$$
u_{1}(x, t)=\frac{K^{\eta}}{2 \epsilon} u(x, t), v_{1}(x, t)=\frac{K^{\eta}}{2 \epsilon}(2 v(x, t)-u(x, t)) .
$$

Then, using (3.15), (3.16), we see that

(i $\left.i_{1}\right) \quad u_{1}(x, t) \geq 0, v_{1}(x, t) \leq 1$ whenever $(x, t) \in\left(\Omega_{T} \backslash \Omega_{T}^{r / K}\right) \cap C_{r}^{-}\left(x_{0}, t_{0}\right)$,

(ii $\left.i_{1}\right) \quad u_{1}(x, t) \geq 1$ whenever $(x, t) \in\left[\partial \Omega^{r / K} \times\left(t_{0}-(r)^{2}, t_{0}\right)\right] \cap C_{r}^{-}\left(x_{0}, t_{0}\right)$,

(iii 1$) \quad v_{1}(x, t) \leq 0$ whenever $(x, t) \in\left[\left(\partial \Omega \cup \partial \Omega^{r / K}\right) \times\left(t_{0}-r^{2}, t_{0}\right)\right] \cap C_{r}^{-}\left(x_{0}, t_{0}\right)$.

Moreover, $u_{1}, v_{1}$ are solutions to $H u=0$ in $\left(\Omega_{T} \backslash \Omega_{T}^{r / K}\right) \cap C_{r}^{-}\left(x_{0}, t_{0}\right)$. In particular, the pair $\left(u_{1}, v_{1}\right)$ satisfies the assumptions stated in Lemma 3.12 with $r$ replaced by $r / K$. Furthermore, by construction we have that

$$
u(x, t)-v(x, t)=\frac{\epsilon}{K^{\eta}}\left(u_{1}(x, t)-v_{1}(x, t)\right) \geq 0,
$$

whenever $(x, t) \in \Omega_{T}^{r / K^{2}} \cap C_{r / K}^{-}\left(x_{0}, t_{0}\right)$. Hence, by iteration of this argument we see that we can construct functions $u_{j}$ and $v_{j}$, for $j=1,2, \ldots$, such that

$$
u(x, t)-v(x, t)=\left(\frac{\epsilon}{K^{\eta}}\right)^{j}\left(u_{j}(x, t)-v_{j}(x, t)\right) \geq 0
$$

whenever $(x, t) \in\left(\Omega_{T} \backslash \Omega_{T}^{r / K^{j+1}}\right) \cap C_{r / K^{j}}^{-}\left(x_{0}, t_{0}\right)$. As a consequence we obtain that

$$
u(x, t)-v(x, t) \geq 0 \text { whenever }(x, t) \in I\left(x_{0}, t_{0}\right),
$$

where $I\left(x_{0}, t_{0}\right)=\bigcup_{j=1}^{\infty} \Omega_{T}^{r / K^{j}} \cap C_{r / K^{j-1}}^{-}\left(x_{0}, t_{0}\right)$. Finally, for arbitrary $\left(\hat{x}_{0}, \hat{t}_{0}\right) \in \Omega_{T} \cap$ $C_{r}^{-}\left(x_{0}, t_{0}\right)$ one can choose $\left(\tilde{x}_{0}, \tilde{t}_{0}\right) \in S_{T}$ such that $d_{p}\left(\hat{x}_{0}, \hat{t}_{0}, S_{T}\right)=d_{p}\left(\hat{x}_{0}, \hat{t}_{0}, \tilde{x}_{0}, \tilde{t}_{0}\right)=$ $d\left(\hat{x}_{0}, \tilde{x}_{0}\right)$. Then $\left(\hat{x}_{0}, \hat{t}_{0}\right) \in I\left(\tilde{x}_{0}, \tilde{t}_{0}\right)$ and $d\left(x_{0}, \tilde{x}_{0}\right)<r$, i.e.,

$\left(\Omega_{T} \backslash \Omega_{T}^{r}\right) \cap C_{K r}^{-}\left(\tilde{x}_{0}, \tilde{t}_{0}\right) \subset\left(\Omega_{T} \backslash \Omega_{T}^{r}\right) \cap C_{(K+2) r}^{-}\left(x_{0}, t_{0}\right)$, $\partial_{p}\left(\left(\Omega_{T} \backslash \Omega_{T}^{r}\right) \cap C_{K r}^{-}\left(\tilde{x}_{0}, \tilde{t}_{0}\right)\right) \cap \partial_{p} \Omega_{T}^{r} \subset \partial_{p}\left(\left(\Omega_{T} \backslash \Omega_{T}^{r}\right) \cap C_{(K+2) r}^{-}\left(x_{0}, t_{0}\right)\right) \cap \partial_{p} \Omega_{T}^{r}$. 
Hence, by replacing $K$ with $K+2$ in the original assumptions and repeating the proof up to here with $\left(\tilde{x}_{0}, \tilde{t}_{0}\right)$ instead of $\left(x_{0}, t_{0}\right)$, we can conclude that $u(x, t)-$ $v(x, t) \geq 0$ on $I\left(\tilde{x}_{0}, \tilde{t}_{0}\right)$ and in particular, $u\left(\hat{x}_{0}, \hat{t}_{0}\right)-v\left(\hat{x}_{0}, \hat{t}_{0}\right) \geq 0$. Since $\left(\hat{x}_{0}, \hat{t}_{0}\right) \in$ $\Omega_{T} \cap C_{r}^{-}\left(x_{0}, t_{0}\right)$ is arbitrary we can hence conclude that $u-v \geq 0$ on $\Omega_{T} \cap$ $C_{r}^{-}\left(x_{0}, t_{0}\right)$. In particular, to complete the proof of Lemma 3.12 we are only left with proving the claim in (3.14).

To do this we proceed as follows. Let $\hat{K}$ be as in the statement of Lemma 3.3 and let $\Lambda \gg 1$ be given. Assume that $K \gg \Lambda \hat{K}$. Given $x_{0} \in \partial \Omega$, according to Lemma 2.8 we can find a set $U$ such that

$$
B_{d}\left(x_{0}, \Lambda \hat{K} r\right) \subset U \subset B_{d}\left(x_{0},(\Lambda+1) \hat{K} r\right),
$$

and such that we can solve the Dirichlet problem (2.11) in

$$
\Omega_{T} \cap\left[U \times\left(t_{0}-16 t^{2}, t_{0}\right)\right] .
$$

Furthermore, we choose $\tilde{x}_{0} \in \Omega$ and $\Lambda$ so that $\tilde{x}_{0} \in \partial B_{d}\left(x_{0}, \Lambda \hat{K} r\right)$ and $B_{d}\left(\tilde{x}_{0}, 2 \hat{K} r\right) \subset$ $\Omega$. We note that, since $\Omega$ is an NTA domain, this can always be accomplished by choosing $\Lambda$ large enough. We next introduce an auxiliary function $\tilde{u}$ as follows. We let $\tilde{u}$ be such that $H \tilde{u}=0$ in $\Omega_{T} \cap\left[U \times\left(t_{0}-16 t^{2}, t_{0}\right)\right], \tilde{u}=1$ on $\partial_{p}\left(\Omega_{T} \cap\left[U \times\left(t_{0}-16 t^{2}, t_{0}\right)\right]\right) \cap C_{\hat{K} r, 2 r}^{-}\left(\tilde{x}_{0}, t_{0}-4 r^{2}\right)$ and $\tilde{u}=0$ on the rest of $\partial_{p}\left(\Omega_{T} \cap\left[U \times\left(t_{0}-16 t^{2}, t_{0}\right)\right]\right)$. We then have $0 \leq \tilde{u} \leq 1$, and $\tilde{u} \leq u$ where $u$ and $\tilde{u}$ are both defined. Also, $\tilde{u}$ is not identical to 1 in $\Omega_{T} \cap\left[U \times\left(t_{0}-16 t^{2}, t_{0}\right)\right]$.

Let $D=U \cap B_{d}\left(\tilde{x}_{0}, \hat{K} r\right)$ and define $\hat{u}=1-\tilde{u}$ in $D \times\left(t_{0}-8 r^{2}, t_{0}-4 r^{2}\right)$. Then $\hat{u}$ satisfies $H \hat{u}=0$ in $D \times\left(t_{0}-8 r^{2}, t_{0}-4 r^{2}\right), \hat{u} \leq 0$ on $\partial_{p}\left(D \times\left(t_{0}-\right.\right.$ $\left.\left.8 r^{2}, t_{0}-4 r^{2}\right)\right) \backslash \partial_{p} C_{\hat{K} r, 2 r}^{-}\left(\tilde{x}_{0}, t_{0}-4 r^{2}\right)$ and $\sup _{D \times\left(t_{0}-8 r^{2}, t_{0}-4 r^{2}\right)} \hat{u}>0$. Because of the construction of $U$, there exists $\hat{x}_{0} \in B_{d}\left(\tilde{x}_{0}, \hat{K} r\right)$ and $\rho$ such that $B_{d}\left(\hat{x}_{0}, \rho\right) \subset$ $B_{d}\left(\tilde{x}_{0}, r\right), B_{d}\left(\hat{x}_{0}, \rho\right) \cap D=\varnothing$ and $\hat{M}^{-1} r<\rho<r$ for some $\hat{M}$ independent of $r$. We can now apply Lemma 3.3 to conclude that there exists a constant $\theta, 0<\theta<1$, independent of $r$, such that

$$
\sup _{\left(D \times\left(t_{0}-8 r^{2}, t_{0}-4 r^{2}\right)\right) \cap C_{r}^{-}\left(\tilde{x}_{0}, t_{0}-4 r^{2}\right)} \hat{u} \leq \theta \sup _{D \times\left(t_{0}-8 r^{2}, t_{0}-4 r^{2}\right)} \hat{u} \leq \theta .
$$

In particular, by continuity we see from (3.17) that

$$
u\left(\tilde{x}_{0}, t_{0}-4 r^{2}\right) \geq \tilde{u}\left(\tilde{x}_{0}, t_{0}-4 r^{2}\right) \geq 1-\theta>0 .
$$

Furthermore, using (3.18), the Harnack inequality and Lemma 3.2 we see that

$$
1-\theta \leq c u\left(A_{r}^{-}\left(x_{0}, t_{0}\right)\right) \leq c^{2} r^{\gamma} u(x, t) d_{p}\left(x, t, S_{T}\right)^{-\gamma}
$$

whenever $(x, t) \in \Omega_{T} \cap C_{r}^{-}\left(x_{0}, t_{0}\right)$. Obviously this gives (3.14) with $\eta=\gamma$ and $2 \epsilon=(1-\theta) / c^{2}$. This completes the proof. 


\section{Proof of Theorem 1.1 and Theorem 1.2}

The purpose of this section is proving Theorems 1.1 and 1.2.

\subsection{Proof of Theorem 1.1}

To begin the proof we let $0<\delta \ll \sqrt{T}$ be a fixed constant, we let $\left(x_{0}, t_{0}\right) \in S_{T}, \delta^{2} \leq$ $t_{0} \leq T-\delta^{2}$, and we assume that $r<\min \left\{r_{0} / 2, \sqrt{\left(T-t_{0}-\delta^{2}\right) / 4}, \sqrt{\left(t_{0}-\delta^{2}\right) / 4}\right\}$. For $\hat{r}>0$ we define

$$
f(\hat{r})=\hat{r}^{-\gamma} \sup _{\Omega_{T} \cap C_{2 \hat{r}}^{-}\left(x_{0}, t_{0}\right)} u(x, t)
$$

where $\gamma$ is the constant appearing in Lemma 3.1. Furthermore, we let

$$
\rho=\max \{\hat{r}: r \leq \hat{r} \leq \delta, f(\hat{r}) \geq f(r)\} .
$$

By the definition of $\rho$ in (4.2) we see that

$$
\sup _{\Omega_{T} \cap C_{2 r}^{-}\left(x_{0}, t_{0}\right)} u(x, t) \leq(r / \rho)^{\gamma} \sup _{\Omega_{T} \cap C_{2 \rho}^{-}\left(x_{0}, t_{0}\right)} u(x, t) .
$$

Furthermore, using Lemma 3.2 we see that

$$
u\left(A_{2 \rho}^{-}\left(x_{0}, t_{0}\right)\right) \leq c(\rho / r)^{\gamma} u\left(A_{r}^{-}\left(x_{0}, t_{0}\right)\right) .
$$

In the following we prove that

$$
\sup _{\Omega_{T} \cap C_{2 \rho}^{-}\left(x_{0}, t_{0}\right)} u(x, t) \leq c u\left(A_{2 \rho}^{-}\left(x_{0}, t_{0}\right)\right)
$$

for this particular choice of $\rho$. In fact, combining (4.3), (4.4) and (4.5) we see that

$$
\sup _{\Omega_{T} \cap C_{2 r}^{-}\left(x_{0}, t_{0}\right)} u(x, t) \leq c u\left(A_{r}^{-}\left(x_{0}, t_{0}\right)\right) .
$$

To prove (4.5) we let $K \gg 1$ be given as in Lemma 3.10, and we divide the proof into two cases. First, we assume that $\delta /(2 K)<\rho$. In this case $\rho$ is large and combining Lemma 3.7 and Lemma 3.9 we see that

$$
\sup _{\Omega_{T} \cap C_{2 \rho}^{-}\left(x_{0}, t_{0}\right)} u(x, t) \leq c u\left(A_{2 \rho}^{+}\left(x_{0}, t_{0}\right)\right) \leq c^{2} u\left(A_{2 \rho}^{-}\left(x_{0}, t_{0}\right)\right),
$$

for some $c=c(H, M$, $\operatorname{diam}(\Omega), T, \delta, K), 1 \leq c<\infty$. Hence, the proof is complete in this case. Second, we assume that $r \leq \rho \leq \delta /(2 K)$ and we then first note, by the definition of $\rho$, that $f(2 K \rho) \leq f(\rho)$, i.e.,

$$
\sup _{\Omega_{T} \cap C_{2 \rho}^{-}\left(x_{0}, t_{0}\right)} u \geq(2 K)^{-\gamma} \sup _{\Omega_{T} \cap C_{4 K \rho}^{-}\left(x_{0}, t_{0}\right)} u .
$$


Obviously the above inequality implies

$$
\sup _{\Omega_{T} \cap C_{2 K \rho, 2 \rho}^{-}\left(x_{0}, t_{0}\right)} u \geq(2 K)^{-\gamma} \sup _{\Omega_{T} \cap C_{4 K \rho, 8 \rho}^{-}\left(x_{0}, t_{0}\right)} u,
$$

and hence we can use Lemma 3.10 to conclude that

$$
\sup _{\Omega_{T} \cap C_{4 K \rho}^{-}\left(x_{0}, t_{0}\right) \cap\left\{(x, t): t=t_{0}-64 \rho^{2}\right\}} u \geq c^{-1} \sup _{\Omega_{T} \cap C_{2 K \rho, 2 \rho}^{-}\left(x_{0}, t_{0}\right)} u .
$$

In particular, using if necessary Lemma 3.7, and the Harnack inequality in Theorem 2.2, we can now use (4.8) to conclude (4.5). This completes the proof of (4.5). Furthermore, Theorem 1 now follows readily from (4.5).

\subsection{Proof of Theorem 1.2}

To prove Theorem 1.2 we first establish a few lemmas.

Lemma 4.1. Let $K \gg 1$ be the constant appearing in Lemma 3.12, let $\left(x_{0}, t_{0}\right) \in$ $S_{T}$ and assume that

$$
r<\min \left\{r_{0} /(2 K), \sqrt{\left(T-t_{0}\right) /\left(4 K^{2}\right)}, \sqrt{t_{0} /\left(4 K^{2}\right)}\right\} .
$$

Let $u$ and $v$ be two nonnegative solutions to $H u=0$ in $\Omega_{T}$, and assume that $v=0$ continuously on $\Delta\left(x_{0}, t_{0}, 2 K r\right)$. Then, there exists a constant $c=c\left(H, M, r_{0}\right)$ such that

$$
\sup _{\Omega_{T} \cap C_{r}^{-}\left(x_{0}, t_{0}\right)} \frac{v}{u} \leq c \frac{v\left(A_{K r}^{+}\left(x_{0}, t_{0}\right)\right)}{u\left(A_{K r}^{-}\left(x_{0}, t_{0}\right)\right)} .
$$

Proof. We first note that if we choose $K$ large enough then, since $\left(\Omega_{T} \backslash \Omega_{T}^{r}\right) \cap$ $C_{K r}^{-}\left(x_{0}, t_{0}\right) \subset \Omega_{T} \cap C_{K r}^{-}\left(x_{0}, t_{0}\right)$, we can use Remark 3.8 to conclude that

$$
v(x, t) \leq c_{1} v\left(A_{K r}^{+}\left(x_{0}, t_{0}\right)\right) \text { whenever }(x, t) \in\left(\Omega_{T} \backslash \Omega_{T}^{r}\right) \cap C_{K r}^{-}\left(x_{0}, t_{0}\right) .
$$

Furthermore, by the Harnack inequality we have that

$$
u(x, t) \geq c_{2}^{-1} u\left(A_{K r}^{-}\left(x_{0}, t_{0}\right)\right),
$$

for every $(x, t) \in \partial_{p}\left(\left(\Omega_{T} \backslash \Omega_{T}^{r}\right) \cap C_{K r}^{-}\left(x_{0}, t_{0}\right)\right) \cap \partial_{p} \Omega_{T}^{r}$. For $(x, t) \in\left(\Omega_{T} \backslash \Omega_{T}^{r}\right) \cap$ $C_{K r}^{-}\left(x_{0}, t_{0}\right)$ let

$$
\begin{gathered}
\tilde{v}(x, t)=v(x, t) / v\left(A_{K r}^{+}\left(x_{0}, t_{0}\right)\right), \\
\tilde{u}(x, t)=u(x, t) / u\left(A_{K r}^{-}\left(x_{0}, t_{0}\right)\right), \\
\hat{v}(x, t)=c_{1}^{-1} \tilde{v}(x, t)-c_{2} \tilde{u}(x, t),
\end{gathered}
$$


and

$$
\hat{u}(x, t)=c_{2} \tilde{u}(x, t) .
$$

Then, we can apply Lemma 3.12 with $u, v$ replaced by $\hat{u}, \hat{v}$ to first conclude that $\hat{v}(x, t) \leq \hat{u}(x, t)$, for $(x, t) \in \Omega_{T} \cap C_{r}^{-}\left(x_{0}, t_{0}\right)$, and then that

$$
\begin{aligned}
\frac{v(x, t)}{u(x, t)} & \leq \frac{v\left(A_{K r}^{+}\left(x_{0}, t_{0}\right)\right)}{u\left(A_{K r}^{-}\left(x_{0}, t_{0}\right)\right)} \frac{c_{2}}{c_{1}}\left(\frac{\hat{v}(x, t)}{\hat{u}(x, t)}+1\right) \\
& \leq c_{3} \frac{v\left(A_{K r}^{+}\left(x_{0}, t_{0}\right)\right)}{u\left(A_{K r}^{-}\left(x_{0}, t_{0}\right)\right)}
\end{aligned}
$$

whenever $(x, t) \in \Omega_{T} \cap C_{r}^{-}\left(x_{0}, t_{0}\right)$. This completes the proof of Lemma 4.1.

Lemma 4.2. Let $K \gg 1$ be the constant appearing in Lemma 3.12, let $\left(x_{0}, t_{0}\right) \in$ $S_{T}$ and assume that

$$
r<\min \left\{r_{0} /(2 K), \sqrt{\left(T-t_{0}\right) /\left(4 K^{2}\right)}, \sqrt{t_{0} /\left(4 K^{2}\right)}\right\} .
$$

Let $u$ and $v$ be two nonnegative solutions to $H u=0$ in $\Omega_{T}$, assume that $u=0$ continuously on $S_{T}$, that $v=0$ continuously on $\Delta\left(x_{0}, t_{0}, 4 K r\right)$, and that $u$ and $v$ are not identically zero. Then, the quotient $v / u$ is Hölder continuous on the closure of $\Omega_{T} \cap C_{r}^{-}\left(x_{0}, t_{0}\right)$.

Proof. To prove this lemma we proceed similarly to [20]. Given $(x, t)$ in the closure of $\Omega_{T}$ and $\rho>0$ we define

$$
\omega(x, t, \rho)=\sup _{\Omega_{T} \cap C_{\rho}^{-}(x, t)} \frac{v}{u}-\inf _{\Omega_{T} \cap C_{\rho}^{-}(x, t)} \frac{v}{u} .
$$

Then, to start with, we note that Lemma 4.1 implies that

$$
\omega\left(x_{0}, t_{0}, 2 r\right) \leq 2 c \frac{v\left(A_{K r}^{+}\left(x_{0}, t_{0}\right)\right)}{u\left(A_{K r}^{-}\left(x_{0}, t_{0}\right)\right)} \leq C<\infty .
$$

In the following we let $(x, t)$ be an arbitrary point in $\Omega_{T} \cap C_{r}^{-}\left(x_{0}, t_{0}\right)$ and we consider $0<\rho \leq r$. Let $d:=d(x, \partial \Omega)=d_{p}\left(x, t, S_{T}\right)$. We divide the proof into the cases $\rho \leq d$ and $\rho>d$.

The case $\rho \leq d$. Assume first that, in addition, $\rho \leq d / 2$. We note that we can assume, without loss of generality, that

(i) $0 \leq \frac{v(y, s)}{u(y, s)} \leq 1$, for $(y, s) \in C_{\rho}^{-}(x, t)$,

(ii) $\omega(x, t, \rho)=1$,

(iii) $\frac{v\left(x, t-\rho^{2} / 2\right)}{u\left(x, t-\rho^{2} / 2\right)} \geq \frac{1}{2}$. 
To see this notice that to achieve (i) and (ii) we can replace $v$ by

$$
\hat{v} \equiv \omega(x, t, \rho)^{-1}\left(v-\left(\inf _{\Omega_{T} \cap C_{\rho}^{-}(x, t)} v / u\right) u\right) .
$$

Furthermore, if (iii) does not hold, then we can replace $v$ by $\bar{v} \equiv u-\hat{v} \geq 0$ to achieve (iii). Next, using the Harnack inequality we first see that

$$
v\left(x, t-\rho^{2} / 2\right) \leq c v(y, s), u(y, s) \leq c u\left(x, t+\rho^{2} / 2\right)
$$

whenever $(y, s) \in C_{\rho / 2}^{-}(x, t)$. Moreover, as in the proof of Theorem 1, we derive that

$$
u\left(x, t+\rho^{2} / 2\right) \leq c u\left(x, t-\rho^{2} / 2\right) .
$$

Thus

$$
\frac{1}{2} \leq \frac{v\left(x, t-\rho^{2} / 2\right)}{u\left(x, t-\rho^{2} / 2\right)} \leq c \frac{v(y, s)}{u(y, s)} \leq c,
$$

whenever $(y, s) \in C_{\rho / 2}^{-}(x, t)$, and hence

$$
\omega(x, t, \rho / 2) \leq \tilde{\theta}_{1} \omega(x, t, \rho)
$$

where $\tilde{\theta}_{1}=1-1 /(2 c) \in(0,1)$. Furthermore, iterating the estimate in (4.12) we deduce that

$$
\omega(x, t, \rho) \leq\left(\frac{2 \rho}{d}\right)^{\sigma_{1}} \omega(x, t, d)
$$

whenever $\rho \leq d / 2$ and where $\sigma_{1}=-\log _{2} \tilde{\theta}_{1}$. Obviously this estimate also holds whenever $d / 2<\rho \leq d$.

The case $\rho>d$. Assume first, in addition, that $\rho<r / 2$. Note that $C_{\rho}^{-}(x, t) \subset$ $C_{2 \rho}^{-}\left(\tilde{x}_{0}, t\right)$ for some $\tilde{x}_{0} \in \partial \Omega$ such that $d=d\left(x, \tilde{x}_{0}\right)$. Then by arguing as in the proof in the case $\rho \leq d$, using Lemma 4.1, it follows as in [20] that

$$
\begin{aligned}
\omega(x, t, \rho) & \leq \omega\left(\tilde{x}_{0}, t, 2 \rho\right) \leq\left(\frac{4 K \rho}{r}\right)^{\sigma_{2}} \omega\left(\tilde{x}_{0}, t, r\right) \\
& \leq\left(\frac{4 K \rho}{r}\right)^{\sigma_{2}} \omega\left(x_{0}, t, 2 r\right),
\end{aligned}
$$

for some $\sigma_{2} \in(0,1)$. Obviously (4.14) also holds in the case $r / 2 \leq \rho \leq r$.

Combining (4.13) and (4.14) we see that

$$
\omega(x, t, \rho) \leq\left(\frac{2 \rho}{d}\right)^{\sigma_{1}} \omega(x, t, d) \leq\left(\frac{2 \rho}{d}\right)^{\sigma_{1}}\left(\frac{4 K d}{r}\right)^{\sigma_{2}} \omega\left(x_{0}, t, 2 r\right)
$$


also when $\rho \leq d<r / 2$. Finally, using (4.11) we obtain for some $\sigma_{3} \in(0,1)$

$$
\omega(x, t, \rho) \leq c K\left(\frac{\rho}{r}\right)^{\sigma_{3}} \omega(x, t, 2 r) \leq c K\left(\frac{\rho}{r}\right)^{\sigma_{3}} C,
$$

whenever $\rho \leq d<r$. Combining these estimates completes the proof of the lemma.

Proof of Theorem 1.2. The interior case is straightforward since both $u$ and $v$ are Hölder continuous and since we only consider solutions which are nonnegative and not identically zero. Hence, we have that the quotient $v / u$ is Hölder continuous in $\Omega_{T}^{\prime} \subset \subset \Omega_{T} \cap C_{r}^{-}\left(x_{0}, t_{0}\right)$. Then to prove Theorem 1.2 we first assume that $u=0$ continuously on $S_{T}$. In this case, using Lemma 4.2, we see that $v / u$ is Hölder continuous on the closure of $\Omega_{T} \cap C_{r_{1}}^{-}(\hat{x}, \hat{t})$, for some small $r_{1}>0$, whenever $(\hat{x}, \hat{t}) \in S_{T} \cap C_{r}^{-}\left(x_{0}, t_{0}\right)$. Combining this fact with the interior argument we see that $v / u$ is Hölder continuous on the closure of $\Omega_{T} \cap C_{r}^{-}\left(x_{0}, t_{0}\right)$. In the general case we represent $u$ in the form $u=u_{0}+u_{1}$, where $L u=L u_{1}=0$ in $\Omega_{T}, u_{0}=0$, $u_{1}=u$ on $S_{T}$ and $u_{0}=u, u_{1}=0$ on $\Omega \times\{t=0\}$. Then by the argument above we see that $v / u_{0}$ as well as $u_{1} / u_{0}$ are Hölder continuous on the closure of $\Omega_{T} \cap C_{r}^{-}\left(x_{0}, t_{0}\right)$. Using this we can conclude, as

$$
\frac{v}{u}=\frac{v}{u_{0}} \frac{1}{1+\frac{u_{1}}{u_{0}}}
$$

that also $v / u$ is Hölder continuous on the closure of $\Omega_{T} \cap C_{r}^{-}\left(x_{0}, t_{0}\right)$. This completes the proof of Theorem 1.2.

\section{Proof of Theorem 1.3}

The objective of this section is proving Theorem 1.3. With this in mind, we first need to introduce some additional notation. In particular, for $\left(x_{0}, t_{0}\right) \in \mathbb{R}^{n+1}$, $0<r_{1}<r_{2}$, and $K \gg 100$, we define,

$$
\begin{aligned}
\Gamma_{K}^{+}\left(x_{0}, t_{0}, r_{1}, r_{2}\right)= & \left\{(x, t)\left|d\left(x, x_{0}\right) \leq K\right| t-\left.t_{0}\right|^{1 / 2},\right. \\
& \left.r_{1} \leq\left|t-t_{0}\right|^{1 / 2} \leq r_{2}, t>t_{0}\right\} .
\end{aligned}
$$

Furthermore, given $\left(x_{0}, t_{0}\right) \in S_{T}, 0<\rho, \mu \in(0,1)$, and a function $u$, we let

$$
f_{1}^{u}\left(x_{0}, t_{0}, \rho, \mu\right)=\inf _{\left\{(x, t): x \in \Omega^{\mu \rho^{\prime}} \cap B_{d}\left(x_{0}, \rho\right), t=t_{0}+\rho^{2}\right\}} u(x, t)
$$

where $\rho^{\prime}=\min \left\{\rho, r_{0}\right\}$. Similarly, given $\left(x_{0}, t_{0}\right) \in S_{T}$ and a function $u$ we define

$$
f_{2}^{u}\left(x_{0}, t_{0}, \rho, K\right)=\sup _{\left\{(x, t) \in \Omega_{T} \cap \partial_{p} C_{K \rho, \rho}\left(x_{0}, t_{0}\right) \cap\left\{(x, t):\left|t-t_{0}\right|<\rho^{2}\right\}\right\}} u^{-}(x, t),
$$

where $u^{-}(x, t)=-\min \{0, u(x, t)\}$.

To establish Theorem 1.3 we will first prove four lemmas. 
Lemma 5.1. Let $\omega^{(x, t)}$ be the H-parabolic measure at $(x, t) \in \Omega_{T}$. Let $\left(x_{0}, t_{0}\right) \in$ $\partial_{p} \Omega_{T}$ and assume that $r<\min \left\{r_{0} / 2\right\}$. Then, there exists a constant $c=c\left(H, M, r_{0}\right)$, $1 \leq c<\infty$, such that

$$
\omega^{(x, t)}\left(C_{2 r}\left(x_{0}, t_{0}\right) \cap \partial_{p} \Omega_{T}\right) \geq c^{-1},
$$

whenever $(x, t) \in \Omega_{T} \cap C_{r}\left(x_{0}, t_{0}\right)$.

Proof. First, let $\left(x_{0}, t_{0}\right) \in S_{T}$. By Lemma 2.8, we can choose a set $U$ which is regular for the Dirichlet problem and such that

$$
B_{d}\left(x_{0}, 3 r / 2\right) \subset U \subset B_{d}\left(x_{0}, 2 r\right) .
$$

Since $\Omega$ is NTA, there exists a point $A_{r}^{\prime}\left(x_{0}\right) \in \mathbb{R}^{n} \backslash \Omega$ such that

$$
\frac{r}{M}<d\left(A_{r}^{\prime}\left(x_{0}\right), x_{0}\right) \leq r, \quad \text { and } d\left(A_{r}^{\prime}\left(x_{0}\right), \partial \Omega\right)>\frac{r}{M} .
$$

Furthermore, using Lemma 2.8 once again we can also find a set $U^{\prime}$, which is $H$ regular for the Dirichlet problem, such that

$$
B_{d}\left(A_{r}^{\prime}\left(x_{0}\right), r / 4 M\right) \subset U^{\prime} \subset B_{d}\left(A_{r}^{\prime}\left(x_{0}\right), r / 2 M\right) \subset U \backslash \Omega .
$$

Using this notation we let

$$
C:=U \times\left[t_{0}-4 r^{2}, t_{0}+4 r^{2}\right], \quad C^{\prime}:=U^{\prime} \times\left[t_{0}-4 r^{2}, t_{0}+4 r^{2}\right],
$$

and $B=U^{\prime} \times\left\{t_{0}-4 r^{2}\right\}$. We also let $v(x, t)=\omega_{C}^{(x, t)}(B)$ and $v^{\prime}(x, t)=\omega_{C^{\prime}}^{(x, t)}(B)$. By the maximum principle, we have $\omega^{(x, t)}\left(\Delta\left(x_{0}, t_{0}, 2 r\right)\right) \geq v(x, t)$ in $C_{r}\left(x_{0}, t_{0}\right) \cap$ $\Omega_{T}$, and $v(x, t) \geq \tilde{v}(x, t)$ in $C^{\prime}$. By the Harnack principle applied in $C$, we have

$$
\begin{aligned}
\inf _{C_{r}\left(x_{0}, t_{0}\right) \cap \Omega_{T}} \omega^{(x, t)}\left(\Delta\left(x_{0}, t_{0}, 2 r\right)\right) & \geq \inf _{C_{r}\left(x_{0}, t_{0}\right) \cap \Omega_{T}} v(x, t) \\
& \geq c^{-1} v\left(A_{r}^{\prime}\left(x_{0}\right), t_{0}-2 r^{2}\right) \\
& \geq c^{-1} v^{\prime}\left(A_{r}^{\prime}\left(x_{0}\right), t_{0}-2 r^{2}\right) .
\end{aligned}
$$

We can extend the function $v^{\prime}$ to the cylinder $C^{\prime \prime}=U^{\prime} \times\left[t_{0}-5 r^{2}, t_{0}+4 r^{2}\right]$ by setting

$$
v^{\prime}(x, t)=\omega_{C^{\prime \prime}}^{(x, t)}\left(\partial_{p}\left(C^{\prime \prime} \cap\left\{t \leq t_{0}-4 r^{2}\right\}\right)\right),
$$

that is, letting $v^{\prime}=1$ below $B$. We now apply the Harnack inequality to $v^{\prime}$ in $C^{\prime \prime}$ and obtain

$$
v^{\prime}\left(A_{r}^{\prime}\left(x_{0}\right), t_{0}-2 r^{2}\right) \geq c^{-1} v^{\prime}\left(A_{r}^{\prime}\left(x_{0}\right), t_{0}-4 r^{2}\right)=c^{-1},
$$

and we are finished. The case when $\left(x_{0}, t_{0}\right)$ is on the bottom of $\partial_{p} \Omega_{T}$ is similar, but simpler. 
Lemma 5.2. Let $K \gg 1$ be given, $\left(x_{0}, t_{0}\right) \in S_{T}$, and assume that $u$ be a solution to $H u=0$ in $\Omega_{T}$ such that $u \geq 0$ in $\Omega_{T} \cap \Gamma_{K}^{+}\left(x_{0}, t_{0}, \rho_{0}, R\right)$ for some $\rho_{0}$ and $R$ such that $0<2 \rho_{0} \leq R \leq v r_{0}$, where $v>0$ is a fixed constant. Then, for every $\mu \in(0,1)$ there exists a $\gamma_{1}>0$ depending on $H, \mu, K, v$, and $r_{0}$, such that

$$
\inf _{\rho_{0} \leq r \leq 2 \rho_{0}} f_{1}^{u}\left(x_{0}, t_{0}, r, \mu\right) \leq\left(\frac{\rho}{\rho_{0}}\right)^{\gamma_{1}} f_{1}^{u}\left(x_{0}, t_{0}, \rho, \mu\right)
$$

for all $\rho$ such that $0<2 \rho_{0} \leq \rho \leq R$.

Proof. To prove Lemma 5.2 we let $\rho$ satisfy $0<2 \rho_{0} \leq \rho \leq R$. We define

$$
D\left(x_{0}, t_{0}, \rho, \mu\right):=\left\{(x, t): x \in \Omega^{\mu \rho^{\prime}} \cap B_{d}\left(x_{0}, \rho\right), t=t_{0}+\rho^{2}\right\}
$$

and note that we can apply the Harnack inequality to conclude that

$$
\begin{aligned}
f_{1}^{u}\left(x_{0}, t_{0}, \rho, \mu\right) & =\inf _{D\left(x_{0}, t_{0}, \rho, \mu\right)} u(x, t) \\
& \geq 2^{-\gamma_{1}} \sup _{D\left(x_{0}, t_{0}, \rho / 2, \mu\right)} u(x, t) \geq 2^{-\gamma_{1}} f_{1}^{u}\left(x_{0}, t_{0}, \rho / 2, \mu\right),
\end{aligned}
$$

for some $\gamma_{1}=\gamma_{1}\left(H, M, r_{0}, \mu\right)>0$. In particular, iterating $k$ times the inequality in (5.4), where $k$ satisfies $2 \rho_{0}>2^{-k} \rho \geq \rho_{0}$, we see that

$$
\begin{aligned}
f_{1}^{u}\left(x_{0}, t_{0}, \rho, \mu\right) & \geq 2^{-k \gamma_{1}} f_{1}^{u}\left(x_{0}, t_{0}, 2^{-k} \rho, \mu\right) \\
& \geq\left(\frac{\rho_{0}}{\rho}\right)^{\gamma_{1}} f_{1}^{u}\left(x_{0}, t_{0}, 2^{-k} \rho, \mu\right) .
\end{aligned}
$$

This latter inequality implies the statement in Lemma 5.2, thus completing the proof.

Lemma 5.3. Let $K \gg 1$ be given, $\left(x_{0}, t_{0}\right) \in S_{T}$, and assume that $u$ is a solution to $H u=0$ in $\Omega_{T}$ such that $u \geq 0$ in $\Omega_{T} \cap \Gamma_{K}^{+}\left(x_{0}, t_{0}, \rho_{0}, R\right)$ for some $\rho_{0}$ and $R$ such that $0<\rho_{0} \leq R$. Furthermore, assume that

$$
u(x, t)=0 \text { whenever }(x, t) \in \partial_{p} \Omega_{T} \backslash C_{\rho_{0} / 2}\left(x_{0}, t_{0}\right) .
$$

Then, there exists $\gamma_{2}>0$, which depends on $H, M, K$ and $r_{0}$, such that

$$
f_{2}^{u}\left(x_{0}, t_{0}, \rho, K\right) \leq\left(\frac{2 \rho_{0}}{\rho}\right)^{\gamma_{2}} f_{2}^{u}\left(x_{0}, t_{0}, \rho_{0}, K\right)
$$

for all $\rho$ such that $0<\rho_{0} \leq \rho \leq R$. Moreover, $\gamma_{2} \rightarrow \infty$ as $K \rightarrow \infty$. 
Proof. By simply using the maximum principle, we first note that since $u=0$ continuously on $\partial_{p} \Omega_{T} \cap\left\{t: t \leq t_{0}-\rho_{0}^{2}\right\}$, we also have that $u \equiv 0$ on $\Omega_{T} \cap$ $\left\{t: t \leq t_{0}-\rho_{0}^{2}\right\}$. Furthermore, again by the maximum principle, applied to $u$ in $\Omega_{T} \backslash \bar{C}_{K \rho, \rho}\left(x_{0}, t_{0}\right)$, we see that as a function of $\rho \in\left[\rho_{0}, R\right]$, the $f_{2}^{u}\left(x_{0}, t_{0}, \rho, K\right)$ decreases, and therefore the conclusion of Lemma 5.3 holds for $\rho \in\left[\rho_{0}, 2 \rho_{0}\right]$. Hence it remains to consider $\rho \in\left(2 \rho_{0}, R\right]$. For such $\rho$ we see that there exists $(\hat{x}, \hat{t}) \in \Omega_{T} \cap \partial_{p} C_{K \rho, \rho}\left(x_{0}, t_{0}\right) \cap\left\{(x, t):\left|t-t_{0}\right|<\rho^{2}\right\}$ such that $f_{2}^{u}\left(x_{0}, t_{0}, \rho, K\right)=$ $u^{-}(\hat{x}, \hat{t})$. Note, in particular, that $d\left(x_{0}, \hat{x}\right)=K \rho$ and that $(\hat{x}, \hat{t}) \in C_{2 \rho}^{+}\left(\hat{x}, t_{0}-\rho^{2}\right)$. Hence,

$$
f_{2}^{u}\left(x_{0}, t_{0}, \rho, K\right)=u^{-}(\hat{x}, \hat{t}) \leq \sup _{\Omega_{T} \cap C_{2 \rho}\left(\hat{x}, t_{0}-\rho^{2}\right)} u^{-}
$$

We claim that that there exists $\hat{K} \gg 1, \hat{K} \ll K, \hat{K}=\hat{K}(H, M)$, such that

$$
\sup _{\Omega_{T} \cap C_{2 \rho}\left(\hat{x}, t_{0}-\rho^{2}\right)} u^{-} \leq \theta \sup _{\Omega_{T} \cap C_{\hat{K} \rho, 2 \rho}\left(\hat{x}, t_{0}-\rho^{2}\right)} u^{-}
$$

for some $\theta \in(0,1)$. This is proved by arguing as in Lemma 3.3, except that the proof is simpler: we only need the function $\Phi_{1}$, and can omit $\Phi_{2}$, since $u^{-}$vanishes on the bottom of $\Omega_{T} \cap C_{\hat{K} \rho, 2 \rho}\left(\hat{x}, t_{0}-\rho^{2}\right)$.

To proceed with the proof of Lemma 5.3 we note that (5.5) and (5.6) imply that

$$
f_{2}^{u}\left(x_{0}, t_{0}, \rho, K\right) \leq \theta \sup _{\Omega_{T} \cap C_{\hat{K} \rho, 2 \rho}\left(\hat{x}, t_{0}-\rho^{2}\right)} u^{-} .
$$

We next note that:

1) the sets $\Omega_{T} \cap C_{\hat{K} \rho, 2 \rho}\left(\hat{x}, t_{0}-\rho^{2}\right)$ and

$$
\Omega_{T} \cap \partial_{p} C_{K \rho_{0}, \rho_{0}}\left(x_{0}, t_{0}\right) \cap\left\{(x, t):\left|t-t_{0}\right|<\rho_{0}^{2}\right\}
$$

are separated by the cylindrical surface

$$
S=\left\{d\left(x_{0}, x\right)=(K-\hat{K}) \rho\right\}=\left\{d\left(x_{0}, x\right)=q K \rho\right\},
$$

where $q=(K-\hat{K}) / K \in[1 / 2,1)$, provided $K \geq 2 \hat{K}$;

2) that

$$
\Omega_{T} \cap \partial_{p} C_{K q \rho, q \rho}\left(x_{0}, t_{0}\right) \cap\left\{(x, t):\left|t-t_{0}\right|<(q \rho)^{2}\right\} \subset S
$$


3) and that $u \geq 0$ in

$$
S \backslash\left(\Omega_{T} \cap \partial_{p} C_{K q \rho, q \rho}\left(x_{0}, t_{0}\right) \cap\left\{(x, t):\left|t-t_{0}\right|<(q \rho)^{2}\right\}\right) .
$$

In particular, by the maximum principle, we obtain that

$$
\begin{aligned}
f_{2}^{u}\left(x_{0}, t_{0}, \rho, K\right) & \leq \theta \sup _{\left\{\Omega_{T} \cap \partial_{p} C_{K q \rho, q \rho}\left(x_{0}, t_{0}\right) \cap\left\{(x, t):\left|t-t_{0}\right|<(q \rho)^{2}\right\}\right\}} u^{-} \\
& =\theta f_{2}^{u}\left(x_{0}, t_{0}, q \rho, K\right)=q^{\gamma_{2}} f_{2}^{u}\left(x_{0}, t_{0}, q \rho, K\right)
\end{aligned}
$$

where $\gamma_{2}=\log _{q} \theta>0$. Next, we choose $k \geq 1$ so that $\rho_{0} \leq q^{k} \rho \leq 2 \rho_{0}$, and by iteration we derive

$$
\begin{aligned}
f_{2}^{u}\left(x_{0}, t_{0}, \rho, K\right) & \leq q^{k \gamma_{2}} f_{2}^{u}\left(x_{0}, t_{0}, q^{k} \rho, K\right) \\
& \leq\left(\frac{2 \rho_{0}}{\rho}\right)^{\gamma_{2}} f_{2}^{u}\left(x_{0}, t_{0}, \rho_{0}, K\right) .
\end{aligned}
$$

Finally, for $K \geq 2 \hat{K}$ we have

$$
\begin{aligned}
\frac{1}{q} & =1+\frac{\hat{K}}{K-\hat{K}} \leq 1+\frac{2 \hat{K}}{K}, \ln q^{-1} \leq \frac{2 \hat{K}}{K}, \\
\gamma_{2} & =\log _{q} \theta \geq \frac{K \ln \left(\theta^{-1}\right)}{2 \hat{K}} \rightarrow \infty \text { as } K \rightarrow \infty .
\end{aligned}
$$

In particular, this completes the proof of Lemma 5.3.

In what follows we let

$$
\Omega_{\left[t_{0}+\rho^{2}, T\right]}=\Omega_{T} \cap\left\{(y, s) \in \mathbb{R}^{n+1} \mid t_{0}+\rho^{2}<s<T\right\} .
$$

For a given Borel set $E \subset \partial_{p} \Omega_{\left[t_{0}+\rho^{2}, T\right]}$, we will denote by $\omega_{\Omega_{\left[t_{0}+\rho^{2}, T\right]}^{(x, t)}}(E)$ the value in $(x, t) \in \Omega_{\left[t_{0}+\rho^{2}, T\right]}$ of the $H$-parabolic measure of $E$.

Lemma 5.4. Let $K \gg 1$ be given, $\left(x_{0}, t_{0}\right) \in S_{T}$, and suppose that $0<2 \rho \leq v r_{0}$, where $v>0$ is a constant. Then, there exist constants $\mu \in(0,1)$ depending on $M$, and $\hat{c}$ depending on $H, M, v$ and $K$, such that

$$
\begin{aligned}
& \omega_{\Omega_{\left[t_{0}+\rho^{2}, T\right]}^{(x, t)}}^{(x)}\left(\Omega_{T} \cap\left(\mathbb{R}^{n} \times\left\{t_{0}+\rho^{2}\right\}\right)\right) \\
& \leq \hat{c} \omega_{\Omega_{\left[t_{0}+\rho^{2}, T\right]}^{(x, t)}}\left(\Omega_{T}^{\mu \rho^{\prime}} \cap\left(B_{d}\left(x_{0}, \rho\right) \times\left\{t_{0}+\rho^{2}\right\}\right)\right),
\end{aligned}
$$

whenever $(x, t) \in \Omega_{T} \cap C_{2 K \rho}\left(x_{0}, t_{0}\right) \cap\left(\mathbb{R}^{n} \times\left\{t_{0}+4 \rho^{2}\right\}\right)$, where $\rho^{\prime}=\min \left(p, r_{0}\right)$. 
Proof. Follows just as the proof of [39, Lemma 4.5]. One also needs to prove the equivalent of [39, Theorem 2.4], which also follows just as in that article. These proofs make use of Lemma 4.1, Lemma 5.1 and the Harnack inequality. We omit the details.

We are finally in a position to establish the main result of this section.

Proof of Theorem 1.3. To start the proof we choose $K \gg 1$ large enough to guarantee that $\gamma_{1}<\gamma_{2}$, where $\gamma_{1}$ and $\gamma_{2}$ are the constants of Lemma 5.2 and Lemma 5.3 respectively. Moreover, for this choice of $\gamma_{1}, \gamma_{2}$, and given the constant in Lemma 5.4, $\hat{c}=\hat{c}(H, M, v, K), 1 \leq \hat{c}<\infty$, we let $\hat{r}=\hat{r}(H, M, v, K)$ be

$$
\text { the smallest } \hat{r} \text { which satisfies } 4^{-\gamma_{1}}(\hat{r} / 4 r)^{\gamma_{2}-\gamma_{1}} \geq \hat{c} \text {. }
$$

Below we will, in the end, distinguish between the cases $v r_{0} \leq \hat{r}$ and $v r_{0}>\hat{r}$. Let $\mu$ be the constant in Lemma 5.4. To prove Theorem 1.3 we intend to prove that there exists a constant $c=c(H, M, v, K)$ such that

$$
u(x, t):=c \omega^{(x, t)}\left(\Delta\left(x_{0}, t_{0}, r\right)\right)-\omega^{(x, t)}\left(\Delta\left(x_{0}, t_{0}, 2 r\right)\right) \geq 0,
$$

whenever $(x, t) \in \Gamma_{K}^{+}\left(x_{0}, t_{0}, 4 r, v r_{0}\right)$. To start the proof of (5.8) we first note, using Lemma 5.1 and the Harnack inequality, that

$$
\omega^{(x, t)}\left(\Delta\left(x_{0}, t_{0}, r\right)\right) \geq \tilde{c}^{-1}
$$

whenever $(x, t) \in \Omega_{T}^{\mu \rho^{\prime}} \cap\left(B_{d}\left(x_{0}, 2 K \rho\right) \times\left\{t_{0}+4 \rho^{2}\right\}\right), 0<2 \rho \leq R \leq v r_{0}$, for some $\tilde{c}=\tilde{c}(H, M, v, K, R), 1 \leq \tilde{c}<\infty$. Let $\hat{c}$ be the constant in Lemma 5.4. Then, using (5.9) and Lemma 5.4 we see that

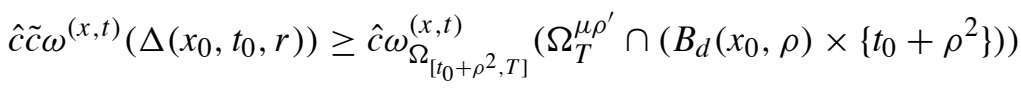

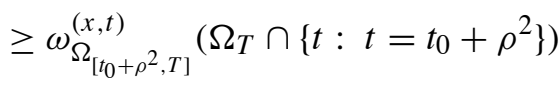

when $(x, t) \in \Omega_{T}^{\mu \rho^{\prime}} \cap\left\{(x, t): x \in B_{d}\left(x_{0}, 2 K \rho\right), t=t_{0}+4 \rho^{2}\right\}$. Note that the first inequality in (5.10) uses (5.9), the trivial inequality $1 \geq \omega\left(x, t, \Omega_{T}^{\mu \rho^{\prime}} \cap\{(x, t)\right.$ : $\left.\left.x \in B_{d}\left(x_{0}, \rho\right), t=t_{0}+\rho^{2}\right\}, \Omega_{\left[t_{0}+\rho^{2}, T\right]}\right)$ and the maximum principle on $\Omega_{T} \cap\{t \geq$ $\left.t_{0}+\rho^{2}\right\}$. Furthermore, let $4 r \leq 2 \rho \leq R \leq v r_{0}$. Then, and this is a simple consequence of the maximum principle,

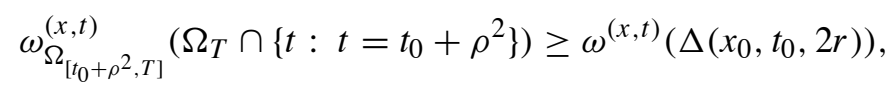

whenever $(x, t) \in \Gamma_{K}^{+}\left(x_{0}, t_{0}, 4 r, v r_{0}\right) \cap\left\{t: t=t_{0}+4 \rho^{2}\right\}$. In particular, combining (5.10) and (5.11) we can conclude that

$$
\hat{c} \tilde{c} \omega^{(x, t)}\left(\Delta\left(x_{0}, t_{0}, r\right)\right) \geq \omega^{(x, t)}\left(\Delta\left(x_{0}, t_{0}, 2 r\right)\right),
$$


whenever $(x, t) \in \Gamma_{K}^{+}\left(x_{0}, t_{0}, 4 r, v r_{0}\right) \cap\left\{t: t=t_{0}+4 \rho^{2}\right\}$. Therefore the function $u$ in (5.8), defined with constant $c=\hat{c} \tilde{c}$, satisfies $u \geq 0$ in $\Gamma_{K}^{+}\left(x_{0}, t_{0}, 4 r, v r_{0}\right)$. In particular, if $v r_{0} \leq \hat{r}$, where $\hat{r}=\hat{r}(H, M, v, K)$ is as in (5.7), then the constant $\tilde{c}$, and hence $c$, can be chosen to only depend on $H, M, v, K$, and we are done. Hence, it only remains to consider the case $v r_{0}>\hat{r}$. However, by arguing as above, we see in this case that there exists $c=c(H, M, v, K)$ such that, if we consider the function $u$ in (5.8) with this $c$, then

(i) $u(x, t) \geq 1$, for $(x, t) \in \Omega_{T}^{\mu \rho^{\prime}} \cap\left\{(x, t): x \in B_{d}\left(x_{0}, \rho\right), t=t_{0}+\rho^{2}\right\}$, for $2 r \leq \rho \leq 4 r$

(ii) $u(x, t) \geq 0$ for $(x, t) \in \Gamma_{K}^{+}\left(x_{0}, t_{0}, 4 r, \hat{r}\right)$.

In the following we prove that (i) and (ii) imply (5.8) for all $(x, t) \in \Gamma_{K}^{+}\left(x_{0}, t_{0}, 4 r, v r_{0}\right)$. To do this we argue by contradiction. Hence, we assume that there exist $\rho>4 r$ such that $u \geq 0$ whenever $(x, t) \in \Gamma_{K}^{+}\left(x_{0}, t_{0}, 4 r, \rho\right)$ and that $u(\hat{x}, \hat{t})<0$ at some point

$$
(\hat{x}, \hat{t}) \in \Omega_{T} \cap\left\{(x, t): x \in B_{d}\left(x_{0}, 2 K \rho\right), t=t_{0}+4 \rho^{2}\right\} \subset \Gamma_{K}^{+}\left(x_{0}, t_{0}, 4 r, 2 \rho\right) .
$$

Let $\omega_{\Omega_{T} \backslash C_{K \rho, \rho}\left(x_{0}, t_{0}\right)}$ denote the $H$-parabolic measure with respect to $\Omega_{T} \backslash C_{K \rho, \rho}\left(x_{0}, t_{0}\right)$. Then, we first note that

$$
\begin{aligned}
u(\hat{x}, \hat{t}) \geq & \int_{\Omega_{T}^{\mu \rho^{\prime}} \cap\left(B_{d}\left(x_{0}, \rho\right) \times\left\{t_{0}+\rho^{2}\right\}\right)} u d \omega_{\Omega_{T} \backslash C_{K \rho, \rho}\left(x_{0}, t_{0}\right)}^{(\hat{x}, \hat{t})} \\
& +\int_{\Omega_{T} \cap \partial_{p} C_{K \rho, \rho}\left(x_{0}, t_{0}\right) \cap\left\{(x, t):\left|t-t_{0}\right|<\rho^{2}\right\}} u d \omega_{\Omega_{T} \backslash C_{K \rho, \rho}\left(x_{0}, t_{0}\right)}^{(\hat{x}, \hat{t})} .
\end{aligned}
$$

Let

$$
E_{1}=\omega_{\Omega_{T} \backslash C_{K \rho, \rho}\left(x_{0}, t_{0}\right)}^{(\hat{x}, \hat{t})}\left(\Omega_{T}^{\mu \rho^{\prime}} \cap\left(B_{d}\left(x_{0}, \rho\right) \times\left\{t_{0}+\rho^{2}\right\}\right)\right),
$$

and

$$
E_{2}=\omega_{\Omega_{T} \backslash C_{K \rho, \rho}\left(x_{0}, t_{0}\right)}^{(\hat{x}, \hat{t})}\left(\Omega_{T} \cap \partial_{p} C_{K \rho, \rho}\left(x_{0}, t_{0}\right) \cap\left\{(x, t):\left|t-t_{0}\right|<\rho^{2}\right\}\right) .
$$

Using (5.13), Lemma 5.2 and Lemma 5.3 we deduce that

$$
u(\hat{x}, \hat{t}) \geq E_{1}\left(\frac{2 r}{\rho}\right)^{\gamma_{1}}-E_{2}\left(\frac{8 r}{\rho}\right)^{\gamma_{2}} .
$$

Furthermore, by the maximum principle and Lemma 5.4 we see that

$$
\begin{aligned}
& \omega_{\Omega_{T} \backslash C_{K \rho, \rho}\left(x_{0}, t_{0}\right)}^{(\hat{t}, \hat{x}}\left(\Omega_{T} \cap \partial_{p} C_{K \rho, \rho}\left(x_{0}, t_{0}\right) \cap\left\{(x, t):\left|t-t_{0}\right|<\rho^{2}\right\}\right)
\end{aligned}
$$

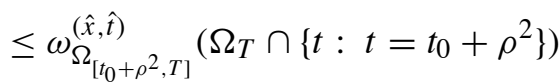

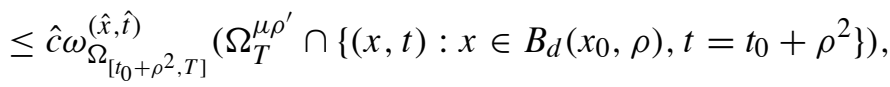


and so $E_{2} \leq \hat{c} E_{1}$. In particular, using that $0>u(\hat{x}, \hat{t})$ and combining (5.14) and (5.15) we can conclude that

$$
4^{-\gamma_{2}}(\rho / 2 r)^{\gamma_{2}-\gamma_{1}}<\hat{c} \leq 4^{-\gamma_{2}}(\hat{r} / 4 r)^{\gamma_{2}-\gamma_{1}} \text {, }
$$

and hence that $2 \rho<\hat{r}$. This implies that $\Gamma_{K}^{+}\left(x_{0}, t_{0}, 4 r, 2 \rho\right) \subset \Gamma_{K}^{+}\left(x_{0}, t_{0}, 4 r, \hat{r}\right)$. Since $(\hat{x}, \hat{t}) \in \Gamma_{K}^{+}\left(x_{0}, t_{0}, 4 r, 2 \rho\right)$ we can therefore conclude from $(B)$ that $u(\hat{x}, \hat{t}) \geq$ 0 . This contradicts our choice of $(\hat{x}, \hat{t})$ and hence (5.8) must be true. This completes the proof of Theorem 1.3.

\section{References}

[1] P. BaUman, Positive solutions of elliptic equations in nondivergence form and their adjoints, Ark. Mat. 22 (1984), 153-173.

[2] A. Bonfiglioli and F. Uguzzoni, Maximum principle and propagation for intrinsicly regular solutions of differential inequalities structured on vector fields, J. Math. Anal. Appl. (2) 322 (2006), 886-900.

[3] J. M. BONY, Principe du maximum, inégalité de Harnack et unicité du problème de Cauchy pour les operateurs elliptique degeneres, Ann. Inst. Fourier (Grenoble) 119 (1969), 277304.

[4] M. Bramanti, L. Brandolini, E. Lanconelli and F. Uguzzoni, Heat kernels for non-divergence operators of Hörmander type, C. R. Math. Acad. Sci. Paris 343 (2006), 463-466.

[5] M. Bramanti, L. Brandolini, E. Lanconelli and F. Uguzzoni, "Non-Divergence Equations Structured on Hörmander Vector Fields: heat Kernels and Harnack Inequalities", Mem. Amer. Math. Soc., Vol. 240, 2010.

[6] L. Caffarelli, E. Fabes, S. Mortola and S. SAlsa, Boundary behavior of nonnegative solutions of elliptic operators in divergence form, Indiana Univ. Math. J. 30 (1981), 621-640.

[7] L. CAPOGNA and N. GAROFALO, Boundary behavior of nonnegative solutions of subelliptic equations in NTA domains for Carnot-Carathéodory metrics, J. Fourier Anal. Appl. 4 (1998), 403-432.

[8] L. Capogna, N. Garofalo and D. M. Nhieu, A subelliptic version of a theorem of Dahlberg for the subelliptic Dirichlet problem, Math. Res. Lett. 5 (1998), 541-549.

[9] L. Capogna, N. Garofalo and D. M. NhiEU, Examples of uniform and NTA domains in Carnot groups, In: "Proceedings on Analysis and Geometry" (Russian) (Novosibirsk Akademgorodok, 1999), Izdat. Ross. Akad. Nauk Sib. Otd. Inst. Mat., Novosibirsk, 2000, $103-121$.

[10] L. Capogna, N. Garofalo and D. M. Nhieu, Properties of harmonic measures in the Dirichlet problem for nilpotent Lie groups of Heisenberg type, Amer. J. Math. 124 (2002), 273-306.

[11] L. Capogna, N. Garofalo and D. M. NhIEU, Mutual absolute continuity of harmonic and surface measure for Hörmander type operators, In: "Perspectives in Partial Differential Equations, Harmonic Analysis and Applications", Proc. Sympos. Pure Math. Amer. Math. Soc., Vol. 79, Providence, RI, 2008, 49-100.

[12] W. L. CHOw, Über systeme von linearen partiellen differentialgleichungen erster ordnug, Math. Ann. 117 (1939), 98-105.

[13] G. CitTI, Wiener estimates at boundary points for Hörmander's operators, Boll. Un. Mat. Ital. B (7) 2 (1988), 667-681. 
[14] D. DANIELLI, Regularity at the boundary for solutions of nonlinear subelliptic equations, Indiana Univ. Math. J. 44 (1995), 269-286.

[15] E. Fabes, N. Garofalo, S. Marin-Malave and S. Salsa, Fatou theorems for some nonlinear elliptic equations, Rev. Mat. Iberoamericana 4 (1988), 227-251.

[16] E. FABES, N. GAROFALO and S. SALSA, A backward Harnack inequality and Fatou theorem for nonnegative solutions of parabolic equations, Illinois J. Math. 30 (1986), 536-565.

[17] E. FABES and C. KENIG, Examples of singular parabolic measures and singular transition probability densities, Duke Math. J. 48 (1981), 845-856.

[18] C. FefFerman and D. H. Phong, Subelliptic eigenvalue problems, Proceedings of the Conference in Harmonic Analysis in Honor of A. Zygmund, Wadsworth Math. Ser., Belmont, CA, (1981), 530-606.

[19] E. FABES and M. SAFONOV, Behaviour near the boundary of positive solutions of second order parabolic equations, J. Fourier Anal. Appl. 3 (1997), 871-882.

[20] E. FABES, M. SAFONOV and Y. YUAN, Behavior near the boundary of positive solutions of second order parabolic equations.II, Trans. Amer. Math. Soc. 351 (1999), 4947-4961.

[21] E. FABES and D. STROoCK, A new proof of Moser's parabolic Harnack inequality using the old ideas of Nash, Arch. Rational Mech. Anal. 96 (1986), 327-338.

[22] N. GAROFALO, Second order parabolic equations in nonvariational forms: boundary Harnack principle and comparison theorems for nonnegative solutions, Ann. Mat. Pura Appl. 138 (1984), 267-296.

[23] N. Garofalo and D. M. Nhieu, Isoperimetric and Sobolev inequalities for CarnotCarathéodory spaces and the existence of minimal surfaces, Comm. Pure Appl. Math. 49 (1996), 1081-1144.

[24] S. HOFMANN and J. LEWIS, The Dirichlet problem for parabolic operators with singular drift term, Mem. Amer. Math. Soc. 151 (2001), 1-113.

[25] H. HörmandER, Hypoelliptic second-order differential equations, Acta Math. 119 (1967), 147-171.

[26] D. JERISON and C. KENIG, Boundary behavior of harmonic functions in nontangentially accessible domains, Adv. Math. 46 (1982), 80-147.

[27] C. KENIG and J. PIPHER, The Dirichlet problem for elliptic operators with drift term, Publ. Mat. 45 (2001), 199-217.

[28] N. V. KRYLOV, sequences of convex functions, and estimates of the maximum of the solution of a parabolic equation, Sibirski Math. Zh. 17 (1976), 226-236.

[29] N. KRYLOV and M. SAFONOV, A property of the solutions of parabolic equations with measurable coefficients, Izv. Akad. Nauk SSSR Ser. Mat. 44 (1980), 161-175.

[30] E. LANCONELli and F. UGUZZONI, Potential analysis for a class of diffusion equations: A Gaussian bounds approach, J. Differential Equations 248 (2010), 2329-2367.

[31] R. MONTI and D. Morbidelli, Non-tangentially accessible domains for vector fields, Indiana Univ. Math. J. 54 (2005), 473-498.

[32] R. Monti and D. Morbidelli, Regular domains in homogeneous groups, Trans. Amer. Math. Soc. 357 (2005), 2975-3011.

[33] I. MunIVE, Boundary behavior of nonnegative solutions of the heat equation in subRiemannian spaces, Potential Anal., advance online publication doi:10.1007/s11118-0119258-5.

[34] K. NYSTRÖM, The Dirichlet problem for second order parabolic operators, Indiana Univ. Math. J. 46 (1997), 183-245.

[35] P. NEGRINI and V. SCORNAZZANI, Wiener criterion for a class of degenerate elliptic operators, J. Differential Equations 166 (1987), 151-167.

[36] A. NAGEL, E. STEIN and S. WAINGER, Balls and metrics defined by vector fields. I. Basic properties, Acta Math. 155 (1985), 103-147.

[37] P. K. RASHEVSKY, Any two points of a totally nonholonomic space may be connected by an admissible line, Uch. Zap. Ped. Inst. im. Liebknechta, Ser. Phys. Math., (Russian) 2 (1938), 83-94. 
[38] S. SALSA, Some properties of nonnegative solution to parabolic differential equations, Ann. Mat. Pura Appl. 128 (1981), 193-206.

[39] M. SAFONOV and Y. YUAN, Doubling properties for second order parabolic equations, Ann. of Math. 150 (1999), 313-327.

[40] F. UGUZZONI, Cone criteria for non-divergence equations modeled on Hörmander vector fields, In: "Subelliptic PDE's and Applications to Geometry and Finance", Lect. Notes Semin. Interdiscip. Mat., 6, Semin. Interdiscip. Mat. (S.I.M.), Potenza, 2007, 227-241.

Department of Mathematics and Mathematical Statistics Umeå University

S-90187 Umeå, Sweden marie.frentz@math.umu.se

Department of Mathematics Purdue University West Lafayette IN 47907-1968, USA garofalo@math.purdue.edu

Department of Mathematics and Mathematical Statistics Umeå University S-90187 Umeå, Sweden elin.gotmark@math.umu.se

Department of Mathematics Purdue University West Lafayette IN 47907-1968, USA imunive@math.purdue.edu

Department of Mathematics Uppsala University S-751 06 Uppsala, Sweden kaj.nystrom@math.uu.se 